\title{
Rigidity of polyhedral surfaces, II
}

\author{
REN GuO \\ FENG LUO
}

\begin{abstract}
We study the rigidity of polyhedral surfaces using variational principles. The action functionals are derived from the cosine laws. The main focus of this paper is on the cosine law for a nontriangular region bounded by three possibly disjoint geodesics. Several of these cosine laws were first discovered and used by Fenchel and Nielsen. By studying the derivative of the cosine laws, we discover a uniform approach to several variational principles on polyhedral surfaces with or without boundary. As a consequence, the work of Penner, Bobenko and Springborn and Thurston on rigidity of polyhedral surfaces and circle patterns are extended to a very general context.
\end{abstract}

52C26, 52B70, 58E30; 51M10, 57Q15

\section{Introduction}

\subsection{Variational principles}

We study geometry of polyhedral surfaces using variational principles in this paper. This can be considered as a continuation of the paper by the second author [9]. By a polyhedral surface we mean an isometric gluing of geometric polygons in $\mathbb{E}^{2}$ (Euclidean plane), $\mathbb{H}^{2}$ (hyperbolic plane) or $\mathbb{S}^{2}$ (the 2-sphere). We emphasize that the combinatorics, ie, the topological cellular decomposition associated to a polyhedral surface, is considered as an intrinsic part of the polyhedral surface. The investigation of the geometry of polyhedral surfaces has a long history. Recent resurgence of interest in this subject is mainly due to the work of William Thurston on geometrization of 3-manifolds and circle packing on surfaces since 1978. Thurston's and Andreev's works on circle packing are nonvariational. The variational approach to circle packing was introduced in a seminal paper by Colin de Verdiére [4]. Since then, many works on variational principles on triangulated surfaces have appeared, for example Brägger [2], Rivin [14], Leibon [8], Bobenko and Springborn [1], Luo [10; 11] and others. A uniform approach to variational principles on triangulated surfaces, based on the derivative of the cosine law for triangles, was proposed in [9]. It is shown in [9] that almost all known variational principles on triangulated surfaces are covered by the cosine law for triangles and right-angled hexagons and their Legendre transformations. 
In [9], compact triangles in spherical, Euclidean and hyperbolic geometry are the building blocks of polyhedral surfaces. Variational principles are established for compact triangles. The goal of this paper is to develop variational principles arising from the cosine laws for hyperbolic polygons bounded by three geodesics. Figure 1 is the list of all ten cases of triangles in the Klein model of the hyperbolic plane. In Figure 2, generalized hyperbolic triangles are drawn in the Poincare model where a horocycle is represented by a circle passing through a vertex. Cosine laws for the cases $(1,1,-1),(-1,-1,1),(-1,-1,-1)$ were discovered in Fenchel and Nielsen's work [5]. R Penner [13] discovered the cosine law for the case $(0,0,0)$ (decorated ideal triangles).

We observe that there is a uniform way to write the cosine laws in all these cases (Lemma 3.1). Furthermore, there is a uniform formula for the derivative cosine laws (Lemma 3.5). From the derivative cosine laws, we are able to find the complete list of localized energy functionals as in [9]. These action functionals provide variational principles for cellular decomposed surfaces. All rigidity results obtained in this paper and the work of Thurston [17], Penner [13] and Bobenko and Springborn [1] can be deduced from those concave energy functionals.

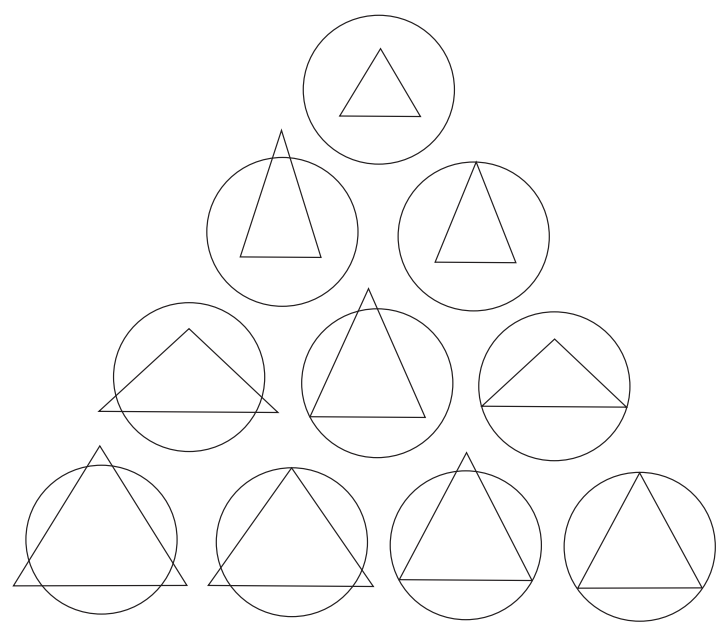

Figure 1: Generalized hyperbolic triangles in Klein model of hyperbolic plane

\subsection{Generalized hyperbolic triangles}

A decorated convex polygon in the hyperbolic plane $\mathbb{H}^{2}$ is a finite area convex polygon $P$ so that each ideal vertex of $P$ is associated with a horodisk centered at the vertex. A generalized hyperbolic triangle (or simply a generalized triangle) $\triangle$ in $\mathbb{H}^{2}$ is a 


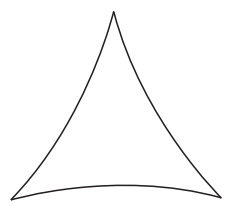

$(1,1,1)$

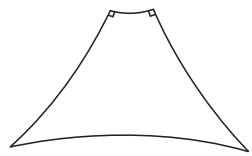

$(1,1,-1)$
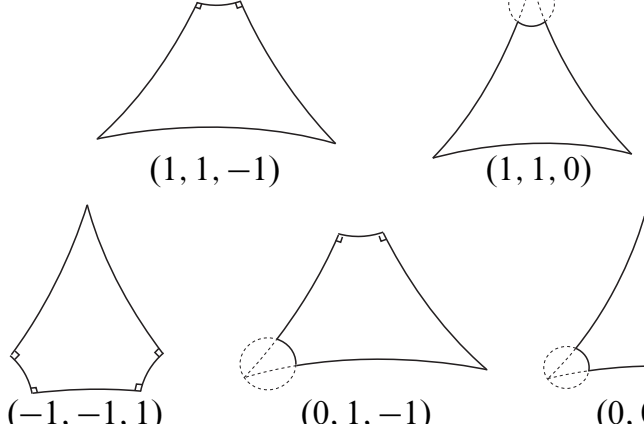

$(0,1,-1)$
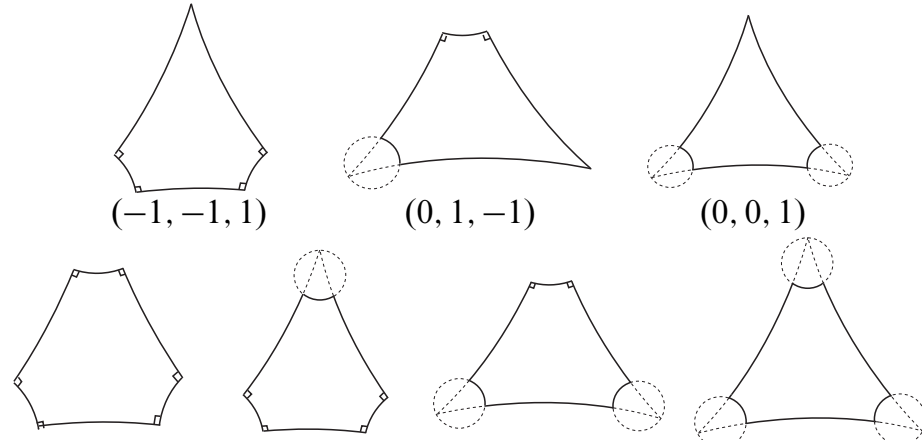

$(-1,-1,-1) \quad(-1,-1,0)$

$(0,0,-1)$

$(0,0,0)$

Figure 2: Generalized hyperbolic triangles in Poincaré model of hyperbolic plane

decorated convex polygon in $\mathbb{H}^{2}$ bounded by three distinct geodesics $L_{1}, L_{2}, L_{3}$ and all other (if any) geodesics $L_{i j}$ perpendicular to both $L_{i}$ and $L_{j}$. The complete list of them is in Figure 2. We call $L_{i} \cap \triangle$ an edge of $\triangle$. In the Klein model of $\mathbb{H}^{2}$, there exists a Euclidean triangle $\widetilde{\triangle}$ in $\mathbb{R}^{2}$ so that each edge of $\widetilde{\triangle}$ corresponds to $L_{1}, L_{2}$ or $L_{3}$.

The vertices of $\widetilde{\Delta}$ are called (generalized) vertices of $\triangle$. Note that if $v$ is a vertex of $\widetilde{\triangle}$ outside $\mathbb{H}^{2} \cup \partial \mathbb{H}^{2}$, then $v$ corresponds to the geodesic $L_{i j}$ perpendicular to the two edges $L_{i}$ and $L_{j}$ adjacent to the vertex $v$. The generalized angle (or simply angle) $a(v)$ at a vertex $v$ of $\triangle$ is defined as follows. Let $L_{i}, L_{j}$ be the edges adjacent to $v$. If $v \in \mathbb{H}^{2}$, then $a(v)$ is the inner angle of $\triangle$ at $v$; if $v \in \partial \mathbb{H}^{2}, a(v)$ is TWICE of the hyperbolic length of the intersection of the associated horocycle with the cusp bounded by $L_{i}$ and $L_{j}$; if $v \notin \mathbb{H}^{2} \cup \partial \mathbb{H}^{2}$, then $a(v)$ is the distance between $L_{i}$ and $L_{j}$. Note that a generalized angle is always positive.

As in Figure 3, for a generalized vertex $u$ of $\triangle$, let $B_{u}=\{u\}$ if $u \in \mathbb{H}^{2}, B_{u}$ be the horodisk at $u$ if $u \in \partial \mathbb{H}^{2}$ and $B_{u}$ is the half plane missing $\triangle$ bounded by $L_{i j}$ if $u \notin \mathbb{H}^{2} \cup \partial \mathbb{H}^{2}$. The generalized edge length (or edge length for simplicity) of $L_{i}$ is 

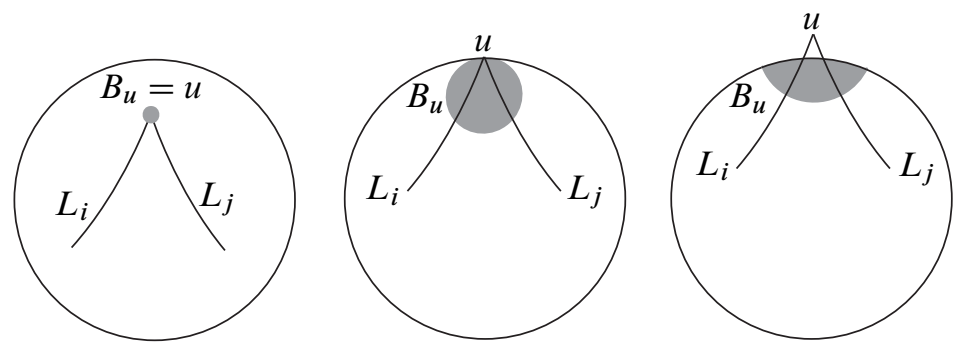

Figure 3: Generalized vertices

defined as follows. The generalized length of the edge $L_{i} \cap \triangle$ with vertices $u, v$ is the distance from $B_{u}$ to $B_{v}$ if $B_{u} \cap B_{v}=\varnothing$ and is the negative of the distance from $\partial B_{u} \cap L_{i}$ to $\partial B_{v} \cap L_{i}$ if $B_{u} \cap B_{v} \neq \varnothing$. Note that generalized edge length may be a negative number. See Figure 4.
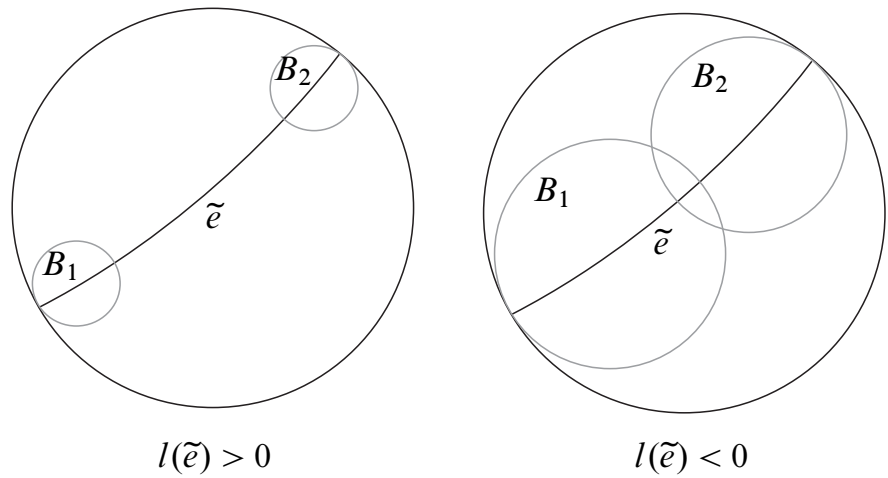

Figure 4: Generalized edge lengths

The cosine law (Lemma 3.1) for generalized triangles relates the generalized angles with the generalized edge lengths. Given a generalized triangle $\triangle$ and a vertex $v$ of $\triangle$, the type $\varepsilon$ of $v$ is defined to be $\varepsilon=1$ if $v \in \mathbb{H}^{2}, \varepsilon=0$ if $v \in \partial \mathbb{H}^{2}$ and $\varepsilon=-1$ if $v \notin \mathbb{H}^{2} \cup \partial \mathbb{H}^{2}$. In this way, generalized triangles are classified into ten types $\left(\varepsilon_{1}, \varepsilon_{2}, \varepsilon_{3}\right)$ where $\varepsilon_{i} \in\{-1,0,1\}$ as in Figure 2.

\subsection{The work of Penner and its generalization}

Suppose $(\tilde{S}, \widetilde{T})$ is a triangulated closed surface $\widetilde{S}$ with the set of vertices $V$, the set of edges $E$. We call $T=\{\sigma-V \mid$ a simplex $\sigma \in \widetilde{T}\}$ an ideal triangulation of the punctured surface $S=\widetilde{S}-V$. We call $V$ ideal vertices (or cusps) of the surface $S$. If the Euler characteristic of $S$ is negative, a decorated hyperbolic metric $(d, r)$ on $S$, 
introduced by Penner [13], is a complete hyperbolic metric $d$ of finite area on $S$ so that each ideal vertex $v$ is assigned a positive number $r_{v}$. Let $T_{c}(S)$ be the Teichmüller space of complete hyperbolic metrics with cusps ends on $S$. Then the decorated Teichmüller space introduced in [13] is $T_{c}(S) \times \mathbb{R}_{>0}^{V}$.

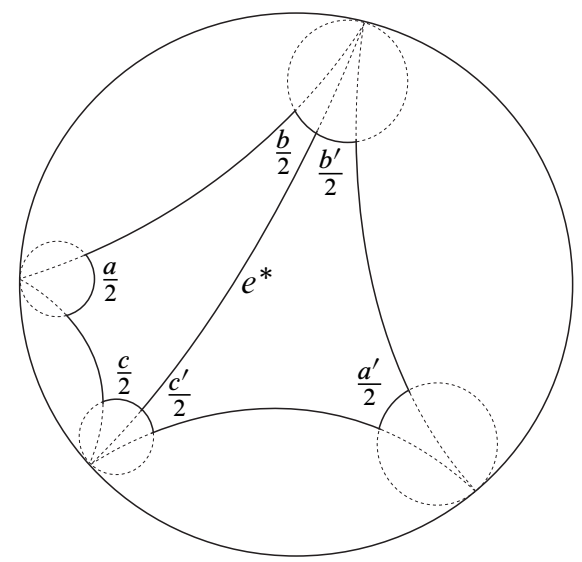

Figure 5: Simplicial coordinates. $a$ is a generalized angle and $\frac{a}{2}$ is the length of the horocyclic arc.

Given a decorated hyperbolic metric $(d, r) \in T_{c}(S) \times \mathbb{R}_{>0}^{V}$, using the ideal triangulation $T$, Penner defined a map $\Psi: T_{c}(S) \times \mathbb{R}_{>0}^{V} \rightarrow \mathbb{R}^{E}$ as follows. Given a metric $(d, r)$, each edge $e \in E$ is isotopic to a complete geodesic $e^{*}$ and each triangle $\sigma$ in $T$ is isotopic to an ideal triangle $\sigma^{*}$ in the metric $d$. Since assigning a positive number $r_{v}$ to each cusp $v$ is the same as associating a horodisk $B$ centered at the cusp so that the length of $\partial B$ is $r_{v}$, we see that each ideal triangle $\sigma^{*}$ is naturally a decorated ideal triangle, ie, a type $(0,0,0)$ generalized hyperbolic triangle. The value of $\Psi(d, r)$ at an edge $e \in E$, is

$$
\Psi(d, r)(e)=\frac{b+c-a}{2}+\frac{b^{\prime}+c^{\prime}-a^{\prime}}{2},
$$

where $a, a^{\prime}$ are the generalized angles facing $e^{*}$, and $b, b^{\prime}, c, c^{\prime}$ are the generalized angles adjacent to $e^{*}$ as labeled in Figure 5.

An edge cycle $\left(e_{1}, t_{1}, e_{2}, t_{2}, \ldots, e_{k}, t_{k}\right)$ in a triangulation $T$ is an alternating sequence of edges $e_{i}$ and faces $t_{i}$ in $T$ so that adjacent faces $t_{i}$ and $t_{i+1}$ share the same edge $e_{i}$ for any $i=1, \ldots, k$ and $t_{k+1}=t_{1}$.

A beautiful theorem proved by Penner is the following. 
Theorem 1.1 (Penner [13]) Suppose $(S, T)$ is an ideally triangulated surface of negative Euler characteristic. Then for any vector $z \in \mathbb{R}_{\geq 0}^{E}$ so that $\sum_{i=1}^{k} z\left(e_{i}\right)>0$ for any edge cycle $\left(e_{1}, t_{1}, \ldots, e_{k}, t_{k}\right)$, there exists a unique decorated complete hyperbolic metric $(d, r)$ on $S$ so that $\Psi(d, r)=z$.

Using the derivative cosine law associated to the decorated ideal triangle and the associated energy function, we generalize Penner's theorem to the following.

Theorem 1.2 Suppose $(S, T)$ is an ideally triangulated surface of negative Euler characteristic. Then Penner's map $\Psi: T_{c}(S) \times \mathbb{R}_{>0}^{V} \rightarrow \mathbb{R}^{E}$ is a smooth embedding whose image is the convex polytope

$$
P(T)=\left\{z \in \mathbb{R}^{E} \mid \sum_{i=1}^{k} z\left(e_{i}\right)>0 \text { for any edge cycle }\left(e_{1}, t_{1}, \ldots, e_{k}, t_{k}\right)\right\} .
$$

We remark that, from the definition, the set $P(T)$ is convex. It is in fact a convex polytope defined by the finite set of linear inequalities $\sum_{i=1}^{k} z\left(e_{i}\right)>0$ for those edge cycles $\left(e_{1}, t_{1}, \ldots, e_{k}, t_{k}\right)$ where each edge appears at most twice (see Luo [11] and Guo [6]).

Results similar to Penner's work have been established recently for hyperbolic cone metric by Leibon [8] and hyperbolic metric with geodesic boundary in [11]. In fact Leibon defined the coordinate $\psi(e)=\frac{b+c-a}{2}+\frac{b^{\prime}+c^{\prime}-a^{\prime}}{2}$, where edge $e$ is shared by two hyperbolic triangles, $a, a^{\prime}$ are the inner angles facing $e$, and $b, b^{\prime}, c, c^{\prime}$ are the inner angles adjacent to $e$. In [11], the coordinate is $\psi(e)=\frac{b+c-a}{2}+\frac{b^{\prime}+c^{\prime}-a^{\prime}}{2}$, where edge $e$ is shared by two hyperbolic right-angled hexagons, $a, a^{\prime}$ are the lengths of boundary arcs $e$, and $b, b^{\prime}, c, c^{\prime}$ are the lengths of boundary arcs adjacent to $e$.

In [9], a one-parameter family of coordinates depending on a parameter $h \in \mathbb{R}$ is introduced for hyperbolic cone metrics and hyperbolic metrics with geodesic boundary. These coordinates generalized the ones in [8] and [11]. In fact, Leibon's coordinate is deformed as

$$
\psi_{h}(e)=\int_{0}^{\frac{b+c-a}{2}} \cos ^{h}(t) d t+\int_{0}^{\frac{b^{\prime}+c^{\prime}-a^{\prime}}{2}} \cos ^{h}(t) d t .
$$

The coordinate in [11] is deformed as

$$
\psi_{h}(e)=\int_{0}^{\frac{a+b-c}{2}} \cosh ^{h}(t) d t+\int_{0}^{\frac{a^{\prime}+b^{\prime}-c^{\prime}}{2}} \cosh ^{h}(t) d t .
$$

These two cases can be written in one formula as

$$
\psi_{h}(e)=\int_{0}^{\frac{a+b-c}{2}} \cos ^{h}(\sqrt{\varepsilon} t) d t+\int_{0}^{\frac{a^{\prime}+b^{\prime}-c^{\prime}}{2}} \cos ^{h}(\sqrt{\varepsilon} t) d t .
$$


Compact hyperbolic triangles correspond to $\varepsilon=1$. Hyperbolic right-angled hexagons correspond to $\varepsilon=-1$. Ideal hyperbolic triangles correspond to $\varepsilon=0$. These $1-$ parameter family of deformations are derived from the associated 1-parameter family of 2-dimensional Schlaefli formulas for the associated generalized hyperbolic triangles. However, there is only one 2-dimensional Schlaefli formula for decorated ideal triangles. This is the reason that Penner's coordinate cannot be deformed.

The relationship between the edge invariant in [11] and Penner's map $\Psi$ was recently established by Mondello [12].

\subsection{Thurston-Andreev's circle packing and its generalizations}

Thurston's work on circle packing can be summarized as follows. Suppose $(\Sigma, T)$ is a triangulated closed surface so that $V, E, F$ are sets of all vertices, edges and triangles in $T$. Fix a map $\Phi: E \rightarrow\left[\frac{\pi}{2}, \pi\right]$. According to Thurston [17], a hyperbolic circle packing metric with intersection angles $\Phi$ is a function $r: V \rightarrow \mathbb{R}_{>0}$ so that the associated edge length function $l: E \rightarrow \mathbb{R}_{>0}$ is defined as follows. In Figure 6, consider a topological triangle with vertices $v_{i}, v_{j}, v_{k}$. One can construct a hyperbolic triangle

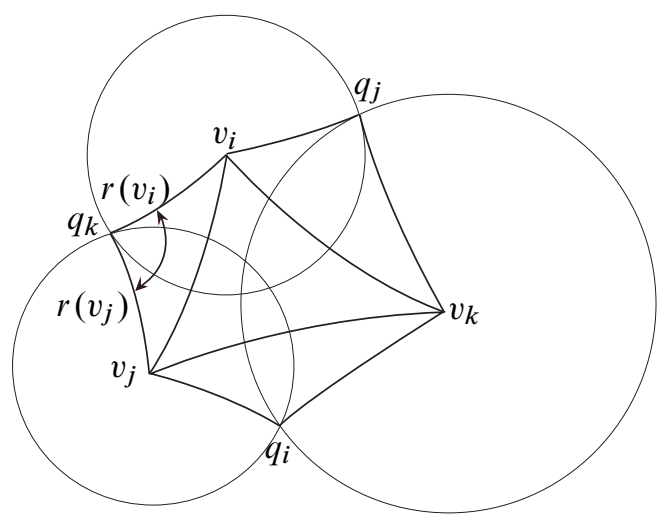

Figure 6: Thurston-Andreev circle packing

$\triangle v_{i} v_{j} q_{k}$ such that the edges $v_{i} q_{k}, v_{j} q_{k}$ have lengths $r\left(v_{i}\right), r\left(v_{j}\right)$ respectively and the angle at $q_{k}$ is $\Phi\left(v_{i} v_{j}\right)$. Let $l\left(v_{i} v_{j}\right)$ be the length of edge $v_{i} v_{j}$ in the hyperbolic triangle $\triangle v_{i} v_{j} q_{k}$, which is a function of $r\left(v_{i}\right), r\left(v_{j}\right)$ and given $\Phi\left(v_{i} v_{j}\right)$ via the cosine law. Similarly, one obtains the edge lengths $l\left(v_{j} v_{k}\right), l\left(v_{k} v_{i}\right)$.

Under the assumption that $\Phi: E \rightarrow\left[\frac{\pi}{2}, \pi\right]$, Thurston observed that lengths $l\left(v_{i} v_{j}\right)$, $l\left(v_{j} v_{k}\right)$ and $l\left(v_{k} v_{i}\right)$ satisfy the triangle inequality for each triangle $\Delta v_{i} v_{j} v_{k}$ in $F$. Thus there exists a hyperbolic polyhedral metric on $(\Sigma, T)$ whose edge length function 
is $l$. Let $K: V \rightarrow \mathbb{R}$ be the discrete curvature of the polyhedral metric, which sends a vertex to $2 \pi$ less the sum of all inner angles at the vertex.

Theorem 1.3 (Thurston [17]) For any closed triangulated surface $(\Sigma, T)$ and any $\Phi: E \rightarrow\left[\frac{\pi}{2}, \pi\right]$, a hyperbolic circle packing metric on $(\Sigma, T)$ is determined by its discrete curvature, ie, the map from $r$ to $K$ is injective. Furthermore, the set of all $K$ 's is an open convex polytope in $\mathbb{R}^{V}$.

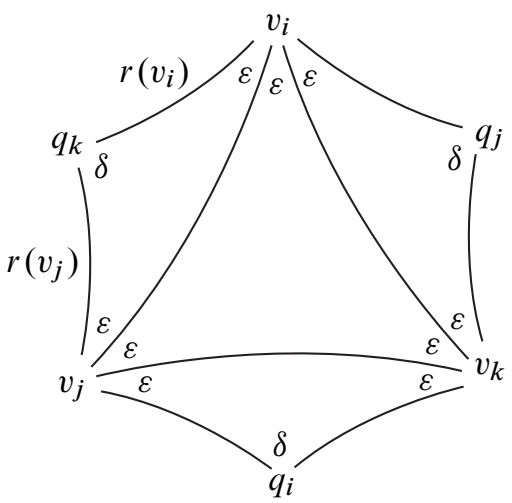

Figure 7: Generalized circle packing

Since there are many other cosine laws available, we may try to use these other cosine laws for generalized triangles of type $(\varepsilon, \varepsilon, \delta)$ instead of type $(1,1,1)$ used by Thurston. To state our result, let us fix the notation once and for all. Let

$$
\begin{aligned}
& I_{\delta}=\left\{\begin{array}{ll}
\mathbb{R}_{>0} & \text { if } \delta=0,-1, \\
(0, \pi] & \text { if } \delta=1 .
\end{array} \quad \stackrel{\circ}{I}_{\delta}= \begin{cases}\mathbb{R}_{>0} & \text { if } \delta=0,-1, \\
(0, \pi) & \text { if } \delta=1,\end{cases} \right. \\
& J_{\sigma}= \begin{cases}\mathbb{R}_{>0} & \text { if } \sigma=1,-1, \\
\mathbb{R} & \text { if } \sigma=0 .\end{cases}
\end{aligned}
$$

A generalized circle packing metric of type $(\varepsilon, \varepsilon, \delta)$ on a triangulated surface $(\Sigma, T)$ with weight $\Phi: E \rightarrow I_{\delta}$ is given by a radius function $r: V \rightarrow J_{\varepsilon \delta}$ so that the edge length function $l: E \rightarrow J_{\varepsilon}$ is obtained from the radius $r$ and weight $\Phi$ by the cosine law applied to the generalized triangle of type $(\varepsilon, \varepsilon, \delta)$. In Figure 7, consider a triangle with vertices $v_{i}, v_{j}, v_{k}$ in the triangulation. One can construct a generalized hyperbolic triangle $\triangle v_{i} v_{j} q_{k}$ of type $(\varepsilon, \varepsilon, \delta)$ such that the edges $v_{i} q_{k}, v_{j} q_{k}$ have lengths $r\left(v_{i}\right)$, $r\left(v_{j}\right)$ respectively and the generalized angle at $q_{k}$ is $\Phi\left(v_{i} v_{j}\right)$. Let $l\left(v_{i} v_{j}\right)$ be the length of edge $v_{i} v_{j}$ in the generalized hyperbolic triangle $\Delta v_{i} v_{j} q_{k}$, which is a function of 
$r\left(v_{i}\right), r\left(v_{j}\right)$ and given $\Phi\left(v_{i} v_{j}\right)$ via the cosine law. Similarly, one obtains the edge lengths $l\left(v_{j} v_{k}\right), l\left(v_{k} v_{i}\right)$.

Depending on $\varepsilon \in\{0,-1,1\}$, the numbers $l\left(v_{i} v_{j}\right), l\left(v_{j} v_{k}\right), l\left(v_{k} v_{i}\right)$ may not be the three edge lengths of a type $(\varepsilon, \varepsilon, \varepsilon)$ triangle. Let $\mathcal{M}_{\varepsilon, \delta}\left(\Phi\left(v_{i} v_{j}\right), \Phi\left(v_{j} v_{k}\right), \Phi\left(v_{k} v_{i}\right)\right)$ be the set of all $\left(r\left(v_{i}\right), r\left(v_{j}\right), r\left(v_{k}\right)\right) \in J_{\varepsilon \delta}^{3}$ such that there exists a type $(\varepsilon, \varepsilon, \varepsilon)$ triangle $\Delta v_{i} v_{j} v_{k}$ with edge lengths $l\left(v_{i} v_{j}\right), l\left(v_{j} v_{k}\right), l\left(v_{k} v_{i}\right)$. Therefore $r$ can only take values in $\mathcal{N}_{\varepsilon, \delta}(\Phi)$ a subspace of $\left(J_{\varepsilon \delta}\right)^{V}$, where $\mathcal{N}_{\varepsilon, \delta}(\Phi)$ is the set of all $r: V \rightarrow J_{\varepsilon \delta}$ such that $\left(r\left(v_{i}\right), r\left(v_{j}\right), r\left(v_{k}\right)\right) \in \mathcal{M}_{\varepsilon, \delta}\left(\Phi\left(v_{i} v_{j}\right), \Phi\left(v_{j} v_{k}\right), \Phi\left(v_{k} v_{i}\right)\right)$, if $v_{i}, v_{j}, v_{k}$ are vertices of a triangle.

The edge length function $l: E \rightarrow J_{\varepsilon}$ produces a polyhedral metric on $(\Sigma, T)$. We define the generalized discrete curvature of the polyhedral metric to be $\widetilde{K}: V \rightarrow \mathbb{R}_{>0}$ sending a vertex to the sum of all generalized angles at the vertex. We remark that the generalized discrete curvature and the discrete curvature in Theorem 1.3 differ by a sign and a constant.

We show that Thurston's circle packing theorem can be generalized to the following six cases corresponding to the generalized triangles of type $(-1,-1,1),(-1,-1,-1)$, $(-1,-1,0),(0,0,1),(0,0,-1),(0,0,0)$ in Figure 8 . More precisely, we list the six cases below.

(a) For the case of $(-1,-1,1)$, where $\Phi: E \rightarrow(0, \pi]$, the edge length $l\left(v_{i} v_{j}\right)$ is obtained from the radii $r\left(v_{i}\right), r\left(v_{j}\right)$ by the cosine law for the hyperbolic pentagon as in Figure 8 (a).

(b) For the case of $(-1,-1,-1)$, where $\Phi: E \rightarrow(0, \infty)$, the edge length $l\left(v_{i} v_{j}\right)$ is obtained from the radii $r\left(v_{i}\right), r\left(v_{j}\right)$ by the cosine law for the right-angled hexagon as in Figure 8 (b).

(c) For the case of $(-1,-1,0)$, where $\Phi: E \rightarrow(0, \infty)$, the edge length $l\left(v_{i} v_{j}\right)$ is obtained from the radii $r\left(v_{i}\right), r\left(v_{j}\right)$ by the cosine law for the hexagon as in Figure 8 (c).

(d) For the case of $(0,0,1)$, where $\Phi: E \rightarrow(0, \pi]$, the edge length $l\left(v_{i} v_{j}\right)$ is obtained from the radii $r\left(v_{i}\right), r\left(v_{j}\right)$ by the cosine law for the pentagon as in Figure 8 (d).

(e) For the case of $(0,0,-1)$, where $\Phi: E \rightarrow(0, \infty)$, the edge length $l\left(v_{i} v_{j}\right)$ is obtained from the radii $r\left(v_{i}\right), r\left(v_{j}\right)$ by the cosine law for the hexagon as in Figure 8 (e).

(f) For the case of $(0,0,0)$, where $\Phi: E \rightarrow(0, \infty)$, the edge length $l\left(v_{i} v_{j}\right)$ is obtained from the radii $r\left(v_{i}\right), r\left(v_{j}\right)$ by the cosine law for the hexagon as in Figure 8 (f). 


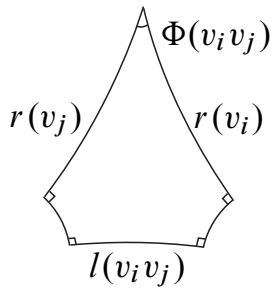

$(-1,-1,1)$

(a)

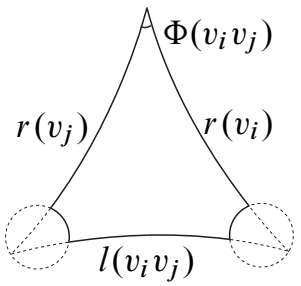

$(0,0,1)$

(d)

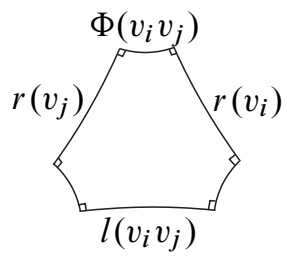

$(-1,-1,-1)$

(b)

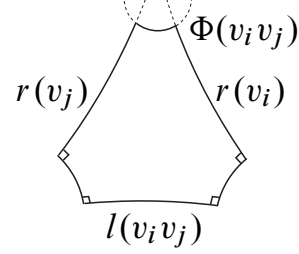

$(-1,-1,0)$

(c)

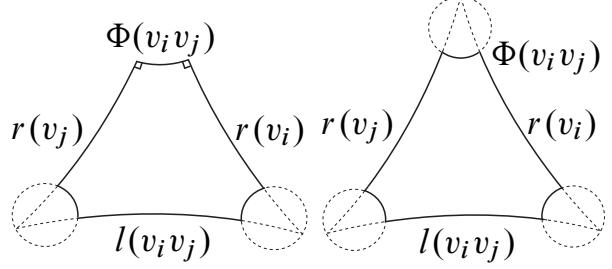

$(0,0,0)$

(f)

Figure 8: Symmetric generalized triangles for generalized circle packing

Theorem 1.4 Given a closed triangulated surface $(\Sigma, T)$ and $\Phi: E \rightarrow I_{\delta}$ in the above six cases, the generalized $(\epsilon, \epsilon, \delta)$ type circle packing metric $r \in \mathcal{N}_{\varepsilon, \delta}(\Phi)$ is determined by its generalized discrete curvature $\tilde{K}: V \rightarrow \mathbb{R}_{>0}$. In particular, the map from $r$ to $\tilde{K}$ is a smooth embedding. Furthermore, the set of all $\tilde{K}$ 's is the space $\mathbb{R}_{>0}^{V}$.

Our method of proof of Theorem 1.4 also produces a new variational proof of the rigidity of circle packing in Thurston's theorem (Theorem 1.3) similar to the proof in [3]. However, unlike the proof in [3] which uses Thurston's geometric argument and Maple, our proof is a straight forward calculation. We are not able to establish Theorem 1.4 for the remaining two cases of $(1,1,-1),(1,1,0)$ in Figure 9.

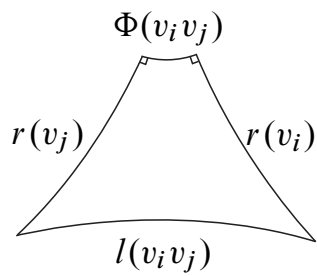

$(1,1,-1)$

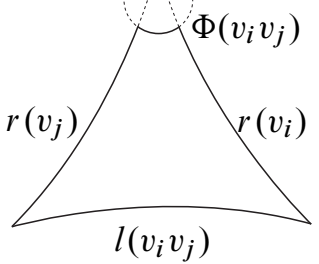

$(1,1,0)$

Figure 9: Generalized circle packing does not work in the two cases. 
Generalized circle patterns have been considered by many authors including Bobenko and Springborn [1], Schlenker [15], Stephenson [16] and others. In particular, Stephenson's question [16, page 331] about disjoint circle patterns motivates us to consider the generalized circle packing of type $(1,1,-1)$.

Furthermore, similar to the work of [3] on discrete curvature flow, for the case of $\varepsilon=-1$ in Theorem 1.4, there exists a corresponding generalized curvature flow. Indeed, let $r_{i}:=r\left(v_{i}\right)$ be the radii at vertex $v_{i} \in V$ and $\widetilde{K}_{i}$ be the generalized discrete curvature at vertex $v_{i} \in V$. The generalized curvature flow is

$$
\frac{d r_{i}(t)}{d t}=-\tilde{K}_{i} \frac{1}{2} e^{r_{i}}-\frac{1}{2} \varepsilon \delta e^{-r_{i}} .
$$

From the proof of Theorem 1.4 in Section 4, we obtain that, when $(\varepsilon, \varepsilon, \delta)$ is one of the six cases in Theorem 1.4, the flow is a negative gradient flow of a strictly concave down function after a change of variables.

\subsection{Bobenko-Springborn's circle pattern and its generalizations}

In [1], Bobenko and Springborn generalized Thurston's circle packing pattern in the case of $\Phi \equiv \pi$ in a different setting. The energy functional in [1] was derived from a discrete integrable system. Let us recall briefly the framework in [1]. See Figure 10 (a). Let $(\Sigma, G)$ be a cellular decomposition of a closed surface with the set of vertices $V$, edges $E$ and 2-cells $F$. The dual cellular decomposition $G^{*}$ has the set of vertices $V^{*}(\cong F)$ so that each $2-$ cell $f$ in $F$ contains exactly one vertex $f^{*}$ in $G^{*}$. If $v$ is a vertex of a 2-cell $f$, we denote it by $v<f$. For all pairs $(v, f)$ where $v<f$, join $v$ to $f^{*}$ by an $\operatorname{arc}$ in $f$, denoted by $\left(v, f^{*}\right)$, so that $\left(v, f^{*}\right)$ and $\left(v^{\prime}, f^{*}\right)$ don't intersect in their interior. Then these $\operatorname{arcs} \bigcup_{(v, f)}\left(v, f^{*}\right)$ decompose the surface $\Sigma$ into a union of quadrilaterals of the form $\left(v, v^{\prime}, f^{*}, f^{*}\right)$ where $v v^{\prime} \in E$, $v<f, v^{\prime}<f^{\prime}$. According to [1], this quadrilateral decomposition of a closed surface arises naturally from integrable systems and discrete Riemann surfaces. Now suppose $\theta: E \rightarrow(0, \pi)$ is given. For $r: V^{*} \rightarrow \mathbb{R}_{>0}$, called a circle pattern metric, and a quadrilateral $\left(v, v^{\prime}, f^{*}, f^{\prime *}\right)$, construct an $\mathbb{E}^{2}$ (or $\mathbb{H}^{2}$ ) triangle $\triangle f^{*} f^{\prime *} v$ so that the length of the edges $v f^{*}, v f^{\prime *}$ are given by $r\left(f^{*}\right), r\left(f^{* *}\right)$ and the angle at $v$ is $\theta\left(v v^{\prime}\right)$.

In this way the quadrilateral $\left(v, v^{\prime}, f^{*}, f^{*}\right)$ is realized in $\mathbb{E}^{2}$ (or $\left.\mathbb{H}^{2}\right)$ as the isometric double of the triangle $\Delta f^{*} f^{\prime *} v$ across the edge $f^{*} f^{\prime *}$. Since the surface $\Sigma$ is a gluing of the quadrilateral $\left(v, v^{\prime}, f^{*}, f^{*}\right)$ along edges, by isometrically gluing these quadrilaterals, one obtains a polyhedral metric on the surface $\Sigma$ with cone points at the vertices $V$ and $V^{*}$. The cone angles at $v \in V$ are prescribed by $\theta$, the only variable curvatures are at $f^{*} \in V^{*}$. Bobenko-Springborn's rigidity result says: 


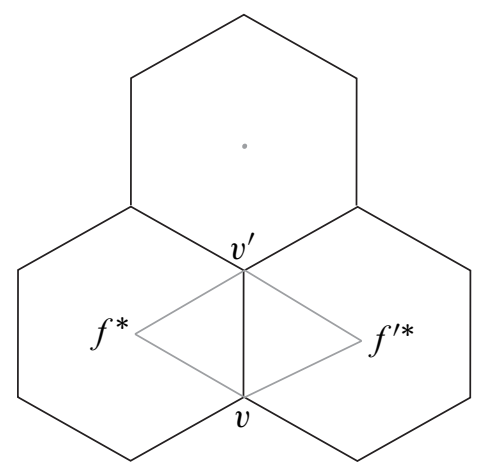

(a)

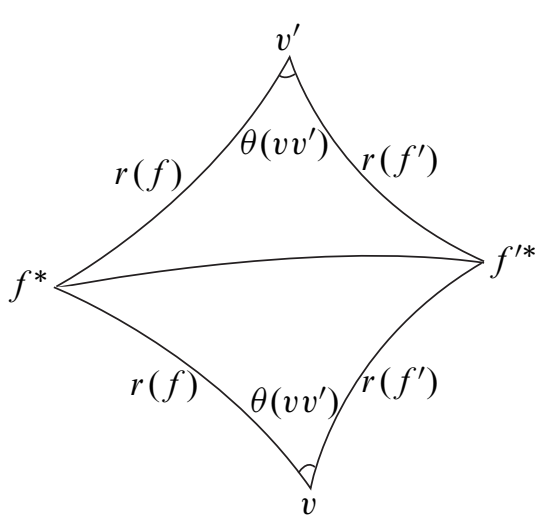

(b)

Figure 10: Bobenko-Springborn's circle pattern

Theorem 1.5 (Bobenko-Springborn [1, Theorems 3 and 4]) For any cell decomposition $(\Sigma, G)$ of a closed surface and any map $\theta: E \rightarrow(0, \pi)$, the circle pattern metric $r: V^{*} \rightarrow \mathbb{R}_{>0}$ is determined by its discrete curvature $K: V^{*} \rightarrow \mathbb{R}$ for hyperbolic polyhedral metrics and is determined up to scaling by $K: V^{*} \rightarrow \mathbb{R}$ for Euclidean polyhedral metrics.

We remark that Bobenko and Springborn [1] proved that the set of all discrete curvatures $K$ forms a convex polytope. In the hyperbolic geometric setting, the essential part of Bobenko and Springborn's construction is to produce the hyperbolic triangle $\triangle f^{*} f^{\prime *} v$ with two prescribed edge lengths and the prescribed angle between the two edges. Since there are eight other generalized hyperbolic triangles of type $(\varepsilon, \varepsilon, \delta)$ as listed in Figure 2, we can use them to produce hyperbolic metrics. Our result below shows that the rigidity phenomena still hold for the other eight cases.

We assume the same setting as in [1] that $(\Sigma, G)$ is a cellular decomposed surface so that $V, E, F$ are the sets of all vertices, edges and 2-cells with dual $\left(\Sigma, G^{*}\right)$. Suppose

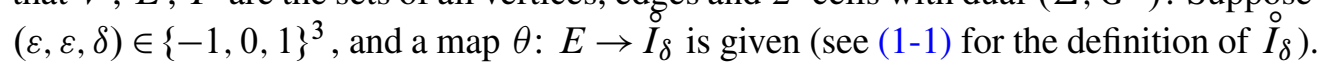

Assume for an $r \in\left(J_{\varepsilon \delta}\right)^{V^{*}}$ and any quadrilateral $\left(v, v^{\prime}, f^{*}, f^{\prime *}\right)$, we can construct a type $(\varepsilon, \varepsilon, \delta)$ generalized hyperbolic triangle $\Delta f^{*} f^{\prime *} v$ so that the lengths of $v f^{*}, v f^{\prime *}$ are $r\left(f^{*}\right), r\left(f^{\prime *}\right)$ respectively and the generalized angle at $v$ is $\theta\left(v v^{\prime}\right)$. Now realize the quadrilateral $\left(v, v^{\prime}, f^{*}, f^{\prime *}\right)$ as the metric double of $\triangle f^{*} f^{*} v$ across the edge $f^{*} f^{\prime *}$. The generalized curvature of the resulting circle pattern is concentrated at the vertices $V^{*}$. It is defined as follows. For $h \in \mathbb{R}$, define the generalized curvature 
$K_{h}: V^{*} \rightarrow \mathbb{R}$ by

$$
K_{h}\left(f^{*}\right)=\sum_{i=1}^{m} 2 \int_{1}^{a_{i}} \rho_{\varepsilon}^{h}(t) d t
$$

where the $a_{i}$ 's are the generalized angles at the vertex $f^{*}$ in the triangle $\triangle f^{*} f^{\prime *} v$ and $\rho_{\varepsilon}(t)=\int_{0}^{t} \cos (\sqrt{\varepsilon} x) d x$ (see the definition in Section 2).

The following theorem is only considering the rigidity of the generalized BobenkoSpringborn circle pattern. We do not have a result addressing the existence, ie, describing the imaging of the map in the following theorem. In the last two subsections about the generalized Penner's map and the generalized Thurston-Andreev circle packing, we established both of rigidity and existence.

Theorem 1.6 Under the setup of generalized circle pattern above, for any $(\varepsilon, \varepsilon, \delta) \in$ $\{-1,0,1\}^{3}$ and $\theta: E \rightarrow \stackrel{\circ}{I}_{\delta}$, the map from $\left(J_{\varepsilon \delta}\right)^{V^{*}}$ to $\mathbb{R}^{V^{*}}$ sending $r$ to $K_{h}$ is a smooth embedding.

Bobenko and Springborn's circle pattern theorem (Theorem 1.5) in the hyperbolic geometry corresponds to $(\varepsilon, \varepsilon, \delta)=(1,1,1)$ and $h=0$ in Theorem 1.6. Bobenko and Springborn [1] also showed that the image of $\{K\}$ is an explicit open convex polytope in $\mathbb{R}^{V^{*}}$. It is an interesting question to investigate the images of $\left\{K_{h}\right\}$ in the generalized setting.

We remark that there is a discrete curvature flow for each case in Theorem 1.6. Let $r_{i}:=r\left(f_{i}^{*}\right)$. The flow is defined by

$$
\frac{d r_{i}(t)}{d t}=-K_{h}\left(f_{i}^{*}\right)\left(\frac{1}{2} e^{r_{i}}-\frac{1}{2} \varepsilon \delta e^{-r_{i}}\right)^{1-h} .
$$

From the proof of Theorem 1.6 in Section 5, we obtain that, for any $(\varepsilon, \varepsilon, \delta) \in$ $\{-1,0,1\}^{3}$, the flow is a negative gradient flow of a strictly concave down function.

Similar situations have been considered before by Hazel. In [7], he considered the flow for cases when $h=0$ and $(\varepsilon, \delta, \varepsilon)=(1,1,1),(1,1,-1)$ or $(1,1,0)$.

Acknowledgment We would like to thank referee for his/her careful reading and very helpful suggestions and comments. 


\section{A proof of Theorem 1.2}

We give a proof of Theorem 1.2 in this section. The proof consists of two parts. In the first part we show that Penner's map $\Psi$ is an embedding. Then we determine its image. As in [11], the rigidity follows from the following well-known fact together with the cosine law for decorated ideal triangles.

Lemma 2.1 If $X$ is an open convex set in $\mathbb{R}^{n}$ and $f: X \rightarrow \mathbb{R}$ is smooth strictly convex, then the gradient $\nabla f: X \rightarrow \mathbb{R}^{n}$ is injective. Furthermore, if the Hessian of $f$ is positive definite for all $x \in X$, then $\nabla f$ is a smooth embedding.

To begin, recall that $(S, T)$ is an ideally triangulated surface with sets of edges, triangles and cusps given by $E, F, V$. We assume that $\chi(S)<0$.

Following Penner [13], we will produce a smooth parametrization of the decorated Teichmüller space $T_{c}(S) \times \mathbb{R}_{>0}^{V}$ by $\mathbb{R}^{E}$ using the edge lengths. From the derivative cosine law for decorated ideal triangles, we will construct a smooth strictly concave down function $H$ on $\mathbb{R}^{E}$ so that its gradient is Penner's map $\Psi$. Then by Lemma 2.1, the map $\Psi: T_{c}(S) \times \mathbb{R}_{>0}^{V} \rightarrow \mathbb{R}^{E}$ is an embedding. To determine the image $\Psi\left(T_{c}(S) \times \mathbb{R}_{>0}^{V}\right)$, we study the degenerations of decorated ideal triangles. The strategy of the proof is the same as that in [11].

\subsection{Penner's length parametrization of $T_{c}(S) \times \mathbb{R}_{>0}^{V}$}

For each decorated hyperbolic metric $(d, r) \in T_{c}(S) \times \mathbb{R}_{>0}^{V}$, where $r=\left(r_{1}, \ldots r_{|V|}\right)$, one replaces each edge $e \in E$ by the geodesic $e^{*}$ in the metric $d$ and constructs for each cusp $v_{i} \in V$ a horocyclic disk $B_{r_{i}}\left(v_{i}\right)$ centered at $v_{i}$ whose circumference $\partial B_{r_{i}}\left(v_{i}\right)$ has length $r_{i}=r\left(v_{i}\right)$. Now, the length coordinate $l_{d, r} \in \mathbb{R}^{E}$ of $(d, r)$ is defined as follows. Given $r: V \rightarrow \mathbb{R}_{>0}$, realize each triangle $\Delta u v w$ in $T$ by a decorated ideal hyperbolic triangle with generalized angles at $u, v, w$ being $r(u), r(v), r(w)$. Then $l_{d, r}(e)$ is the generalized edge length of the edge $e=u v$ in the triangle $\Delta u v w$. In this way, Penner defined a length map

$$
\begin{aligned}
L: T_{c}(S) \times \mathbb{R}_{>0}^{V} & \rightarrow \mathbb{R}^{E} \\
(d, r) & \rightarrow l_{d, r} .
\end{aligned}
$$

Lemma 2.2 (Penner [13]) The length map $L: T_{c}(S) \times \mathbb{R}_{>0}^{V} \rightarrow \mathbb{R}^{E}$ is a diffeomorphism. 
Proof By the cosine law for decorated ideal triangle, the map $L$ satisfies for all $\lambda \in \mathbb{R}_{>0}$

$$
L(d, \lambda r)=L(d, r)-(2 \ln \lambda)(1,1, \ldots, 1) .
$$

Thus, it suffices to deal with those decorated metrics $(d, r)$ so that $r$ are small, ie, $L(d, r) \in \mathbb{R}_{>0}^{E}$. In this case, Penner proved that $L \mid: L^{-1}\left(\mathbb{R}_{>0}^{E}\right) \rightarrow \mathbb{R}_{>0}^{E}$ is a diffeomorphism by a direct geometric construction using isometric gluing of decorated ideal triangles. By (2-1), it follows that $L$ is a diffeomorphism.

\subsection{Penner's map $\Psi$ is a coordinate}

Recall that for a decorated ideal triangle $\Delta$ with edges $e_{1}, e_{2}, e_{3}$ of lengths $l_{1}, l_{2}, l_{3}$ and opposite generalized angles $\theta_{1}, \theta_{2}, \theta_{3}$, the cosine law obtained by Penner [13] says

$$
\frac{e^{l_{i}}}{2}=\frac{2}{\theta_{j} \theta_{k}}, \quad \frac{\theta_{i}^{2}}{4}=e^{l_{i}-l_{j}-l_{k}} .
$$

where $\{i, j, k\}=\{1,2,3\}$. The derivative cosine law expressing $l_{i}$ in terms of $\left(\theta_{1}, \theta_{2}, \theta_{3}\right)$ says

$$
\frac{\partial l_{i}}{\partial \theta_{i}}=0, \quad \frac{\partial l_{i}}{\partial \theta_{j}}=-\frac{1}{\theta_{j}} .
$$

Let $x_{i}=\frac{1}{2}\left(\theta_{j}+\theta_{k}-\theta_{i}\right)$ (or $\left.\theta_{i}=x_{j}+x_{k}\right)$. We call $x_{i}$ the radius invariant at the edge $e_{i}$ in the triangle $\triangle$.

Using the derivative cosine law, we have:

Lemma 2.3 Under the same assumption as above, the differential 1-form $\omega=$ $\sum_{i=1}^{3} x_{i} d l_{i}$ is closed in $\mathbb{R}^{3}$ and its integration $W(l)=\int_{0}^{l} \omega$ is strictly concave down in $\mathbb{R}^{3}$. Furthermore,

$$
\frac{\partial W}{\partial l_{i}}=x_{i}
$$

Proof Consider the matrix $H=\left[\partial l_{a} / \partial x_{b}\right]_{3 \times 3}$. The closeness of $\omega$ is equivalent to that $H$ is symmetric. The strictly concavity of $W$ will be a consequence of the negative definiteness of $H$. We establish these two properties for $H$ as follows. Assume that indices $\{i, j, k\}=\{1,2,3\}$. By definition, $\partial / \partial x_{i}=\partial / \partial \theta_{j}+\partial / \partial \theta_{k}$. It follows from the derivative cosine law that

and

$$
\begin{aligned}
\frac{\partial l_{i}}{\partial x_{i}} & =-\left(\frac{1}{\theta_{j}}+\frac{1}{\theta_{k}}\right), \\
\frac{\partial l_{i}}{\partial x_{j}} & =-\frac{1}{\theta_{k}},
\end{aligned}
$$


which is symmetric in $i, j$. This shows that the matrix $H$ is symmetric. Furthermore, the negative matrix $-H$ is of the form $\left[m_{a b}\right]_{3 \times 3}$ where $m_{a b}=m_{b a}>0$ and $m_{i i}=m_{i j}+m_{i k}$. The determinant of such a matrix can be calculated easily as $4 m_{11} m_{22} m_{33}>0$, and the determinant of a principal $2 \times 2$ submatrix is $m_{i i} m_{j j}-m_{i j}^{2}=\left(m_{i j}+m_{i k}\right)\left(m_{i j}+m_{j k}\right)-m_{i j}^{2}>0$. It follows that $-H$ is positive definite.

By the construction in Section 2.1, it suffices to show that the composition $\widetilde{\Psi}=$ $\Psi \circ L^{-1}: \mathbb{R}^{E} \rightarrow \mathbb{R}^{E}$ is a smooth embedding. The map $\widetilde{\Psi}$ is constructed explicitly as follows. For each $l \in \mathbb{R}^{E}$ and each triangle $\sigma \in F$ realize $\sigma$ by an ideal hyperbolic triangle together with horocycles centered at three vertices so that the generalized edge length of an edge $e$ in $\sigma$ is $l(e)$. Now isometrically glue these ideal hyperbolic triangles along edges so that the horocycles match. The result is a complete finite area hyperbolic metric on the surface $S$ together with a horocycle at each cusp. For each edge $e \in E$, the value $\widetilde{\Psi}(l)(e)$ is equal to $\frac{b+c-a}{2}+\frac{b^{\prime}+c^{\prime}-a^{\prime}}{2}$ where $a, a^{\prime}, b, b^{\prime}, c$, $c^{\prime}$ are generalized angles facing and adjacent to $e$ in Figure 5. Thus

$$
\widetilde{\Psi}(l)(e)=r_{f}(e)+r_{f^{\prime}}(e)
$$

where $f, f^{\prime}$ are the decorated ideal triangles sharing the edge $e$ and $r_{f}(e), r_{f^{\prime}}(e)$ are the radius invariants at the edge $e$ in $f, f^{\prime}$ respectively.

Given a vector $l \in \mathbb{R}^{E}$, define the energy $H(l)$ of $l$ to be

$$
H(l)=\sum_{\{i, j, k\} \in F} W\left(l\left(e_{i}\right), l\left(e_{j}\right), l\left(e_{k}\right)\right)
$$

where the sum is over all triangles $\{i, j, k\}$ in $F$ with edges $e_{i}, e_{j}, e_{k}$. By definition and Lemma 2.3,H: $\mathbb{R}^{E} \rightarrow \mathbb{R}$ is smooth and strictly concave down when Hessian is negative definite. Furthermore, by (2-2) and (2-3),

$$
\frac{\partial H}{\partial l\left(e_{i}\right)}=\widetilde{\Psi}(l)\left(e_{i}\right)
$$

ie, $\nabla H=\widetilde{\Psi}$. It follows from Lemma 2.1 , that $\widetilde{\Psi}: \mathbb{R}^{E} \rightarrow \mathbb{R}^{E}$ is a smooth embedding. Therefore $\Psi$ is a smooth embedding.

\subsection{The image of Penner's map}

Let $\Omega$ be the convex subset of $\mathbb{R}^{E}$ consisting of all $z \in \mathbb{R}^{E}$ such that $\sum_{i=1}^{p} z\left(e_{n_{i}}\right)>0$ whenever $\left(e_{n_{1}}, t_{n_{1}}, e_{n_{2}}, t_{n_{2}}, \ldots, e_{n_{p}}, t_{n_{p}}, e_{n_{1}}\right)$ is an edge cycle. To show that $\widetilde{\Psi}\left(\mathbb{R}^{E}\right)=$ $\Omega$, due to convexity of $\Omega$, it suffices to prove that $\widetilde{\Psi}\left(\mathbb{R}^{E}\right)$ is both open and closed in $\Omega$. 


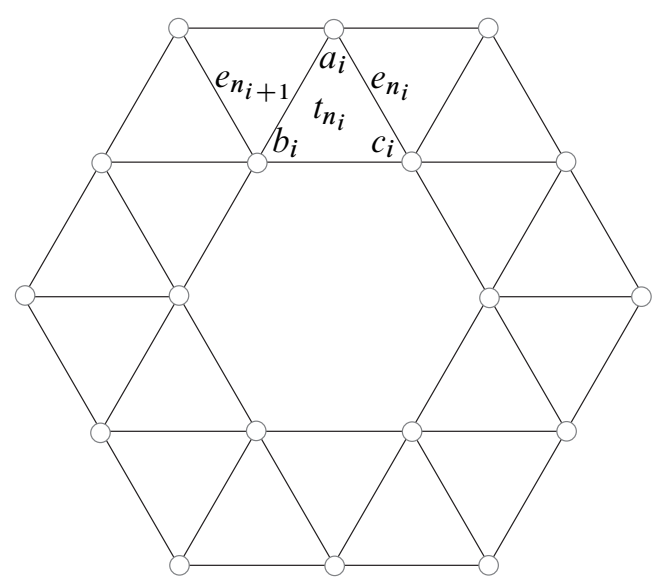

Figure 11: Edge cycles

First to see that $\widetilde{\Psi}\left(\mathbb{R}^{E}\right) \subset \Omega$, take an edge cycle $\left(e_{n_{1}}, t_{n_{1}}, e_{n_{2}}, t_{n_{2}}, \ldots, e_{n_{p}}, t_{n_{p}}, e_{n_{1}}\right)$ as shown in Figure 11 and take $l \in \mathbb{R}^{E}$.

Let the generalized angles in the decorated ideal triangle $t_{n_{i}}$ in the metric $L^{-1}(l)$ be $a_{i}, b_{i}, c_{i}$, where $b_{i}$ faces the edge $e_{n_{i}}, c_{i}$ faces the edge $e_{n_{i+1}}$ and $a_{i}$ is adjacent to $e_{n_{i}}, e_{n_{i+1}}$. Then the contribution to $\sum_{j=1}^{p} \widetilde{\Psi}(l)\left(e_{n_{j}}\right)$ from $e_{n_{i}}, e_{n_{i+1}}$ in triangle $t_{n_{i}}$ is given by $\left(a_{i}+b_{i}-c_{i}\right) / 2+\left(a_{i}+c_{i}-b_{i} / 2\right)=a_{i}$. Thus

$$
\sum_{j=1}^{p} \widetilde{\Psi}(l)\left(e_{n_{j}}\right)=\sum_{j=1}^{p} a_{i}>0
$$

due to $a_{i}>0$ for all $i$. It follows that $\widetilde{\Psi}\left(\mathbb{R}^{E}\right)$ is open in $\Omega$ since $\widetilde{\Psi}: \mathbb{R}^{E} \rightarrow \mathbb{R}^{E}$ was just proved to be an embedding.

It remains to prove that $\widetilde{\Psi}\left(\mathbb{R}^{E}\right)$ is closed in $\Omega$. The closeness of $\widetilde{\Psi}\left(\mathbb{R}^{E}\right)$ in $\Omega$ requires to show that if a sequence $\left\{l^{(m)} \in \mathbb{R}^{E}\right\}_{m=0}^{\infty}$ satisfies $\lim _{m \rightarrow \infty} \widetilde{\Psi}\left(l^{(m)}\right)=z \in \Omega$, then $\left\{l^{(m)}\right\}_{m=0}^{\infty}$ contains a subsequence converging to a point in $\mathbb{R}^{E}$. Given a decorated hyperbolic metric $l^{(m)} \in \mathbb{R}^{E}$ on $(S, T)$ and a generalized angle $\theta$, let $\theta^{(m)} \in \mathbb{R}^{3 F}$ be the generalized angles of the decorated ideal triangles in $(S, T)$ in the metric $l^{(m)}$. By taking a subsequence if necessary, we may assume that $\lim _{m \rightarrow \infty} \widetilde{\Psi}\left(l^{(m)}\right)$ converges in $[-\infty, \infty]^{E}$ and that for each angle $\theta_{i}$, the $\operatorname{limit}_{m \rightarrow \infty} \theta_{i}^{(m)}$ exists in $[0, \infty]$.

Lemma 2.4 For all $i, \lim _{m \rightarrow \infty} \theta_{i}^{(m)} \in[0, \infty)$. 
Proof If otherwise, suppose that $\lim _{m \rightarrow \infty} \theta_{1}^{(m)}=\infty$ for some angle $\theta_{1}$. Let $e_{1}, e_{2}$ be the edges adjacent to the angle $\theta_{1}$ in the triangle $t$. Take an edge cycle $\left(e_{n_{1}}, t_{n_{1}}, e_{n_{2}}, t_{n_{2}}, \ldots, e_{n_{p}}, t_{n_{p}}, e_{n_{1}}\right)$ which contains $\left(e_{1}, t, e_{2}\right)$ as a part. Then by the calculation as in (2-4),

$$
\sum_{i=1}^{p} z\left(e_{n_{i}}\right)=\lim _{m \rightarrow \infty} \sum_{i=1}^{p} \widetilde{\Psi}\left(l^{(m)}\right)\left(e_{n_{i}}\right) \geq \lim _{m \rightarrow \infty} \theta_{1}^{(m)}=\infty .
$$

This contradicts the assumption that $z \in \Omega$.

Now, we finish the proof by contradiction as follows. If $\lim _{m \rightarrow \infty} l^{(m)}$ were not in $\mathbb{R}^{E}$, there would exist an edge $e \in E$ so that $\lim _{m \rightarrow \infty} l^{(m)}(e)= \pm \infty$. Let $t$ be a triangle adjacent to $e$. Let $\theta_{1}^{(m)}, \theta_{2}^{(m)}$ be the generalized angles in $t$ adjacent to $e$ in the metric $l^{(m)}$. By the cosine law,

$$
\exp \left(l^{(m)}(e)\right)=\frac{4}{\theta_{1}^{(m)} \theta_{2}^{(m)}}
$$

and $\theta_{1}^{(m)}, \theta_{2}^{(m)} \in(0, \infty)$

Case 1 If $\lim _{m \rightarrow \infty} l^{(m)}(e)=-\infty$, then $\lim _{m \rightarrow \infty} \exp \left(l^{(m)}(e)\right)=0$. By (2-5), one of $\lim _{m \rightarrow \infty} \theta_{i}^{(m)}$ must be $\infty$. But this contradicts Lemma 2.4.

Case 2 If $\lim _{m \rightarrow \infty} l^{(m)}(e)=\infty$, then $\lim _{m \rightarrow \infty} \exp \left(l^{(m)}(e)\right)=\infty$. Since Lemma 2.4 shows that $\theta_{1}^{(m)}$ and $\theta_{2}^{(m)}$ are bounded, by (2-5), one of the limits $\lim _{m \rightarrow \infty} \theta_{i}^{(m)}$ must be zero for $i=1$ or 2 . Say $\lim _{m \rightarrow \infty} \theta_{1}^{(m)}=0$. Let $e_{1}$ be the other edge in the triangle $t$ so that $e_{1}, e$ are adjacent to the generalized angle $\theta_{1}$. Let $\theta_{3}$ be the third angle in $t$, facing $e$. Then by the cosine law that $\exp \left(l^{(m)}\left(e_{1}\right)\right)=4 /\left(\theta_{1}^{(m)} \theta_{3}^{(m)}\right)$ and Lemma 2.4 on the boundedness of $\theta_{3}^{(m)}$, we conclude that $\lim _{m \rightarrow \infty} l^{(m)}\left(e_{1}\right)=\infty$. To summarize, from $\lim _{m \rightarrow \infty} l^{(m)}(e)=\infty$ and any triangle $t$ adjacent to $e$, we conclude that there is an edge $e_{1}$ and an angle $\theta_{1}$ adjacent to $e, e_{1}$ in $t$ so that $\lim _{m \rightarrow \infty} l^{(m)}\left(e_{1}\right)=\infty$ and $\lim _{m \rightarrow \infty} \theta_{1}^{(m)}=0$.

Applying this procedure to $e_{1}$ and the triangle $t_{1}$ adjacent to $e_{1}$ from the side other than the side that $t$ lies. We obtain the next angle, say $\theta_{2}$ and edge $e_{2}$ in $t_{1}$ so that $\lim _{m \rightarrow \infty} l^{(m)}\left(e_{2}\right)=\infty$ and $\lim _{m \rightarrow \infty} \theta_{2}^{(m)}=0$. Since there are only finite number of edges and triangles, this procedure will produce an edge cycle $\left(e_{n_{1}}, t_{n_{1}}, e_{n_{2}}, t_{n_{2}}, \ldots, e_{n_{k}}, t_{n_{k}}, e_{n_{1}}\right)$ in $T$ so that 
(i) $\lim _{m \rightarrow \infty} l^{(m)}\left(e_{n_{i}}\right)=\infty$ for each $i$,

(ii) $\lim _{m \rightarrow \infty} \theta_{i}^{(m)}=0$ for each $i$, where $\theta_{i}$ is the angle in triangle $t_{n_{i}}$ adjacent to $e_{n_{i}}$ and $e_{n_{i+1}}$.

By (2-4),

$$
\sum_{i=1}^{k} z\left(e_{n_{i}}\right)=\lim _{m \rightarrow \infty} \sum_{i=1}^{k} \widetilde{\Psi}\left(l^{(m)}\right)\left(e_{n_{i}}\right)=\lim _{m \rightarrow \infty} \sum_{i=1}^{k} \theta_{i}^{(m)}=0 .
$$

This contradicts the assumption that $z \in \Omega$.

\section{The derivative cosine law}

We give a unified approach to all cosine laws and sine laws in this section. The derivatives of the cosine laws are also determined. Most of the proofs are straightforward checking and will be delayed to Appendix B.

Assume that a generalized hyperbolic triangle of type $\left(\varepsilon_{1}, \varepsilon_{2}, \varepsilon_{3}\right) \in\{-1,0,1\}^{3}$ has generalized angles $\theta_{1}, \theta_{2}, \theta_{3}$ and opposite generalized edge lengths $l_{1}, l_{2}, l_{3}$. There are ten different types of generalized hyperbolic triangles as shown in Figure 2 . The relationships between $l_{i}^{\prime} \mathrm{s}$ and $\theta_{i}^{\prime} \mathrm{s}$ are expressed in the cosine law and the sine law. To state them, we introduce the two functions $\rho_{\varepsilon}, \tau_{s}$ depending on $\varepsilon, \theta, s, l \in \mathbb{R}$ :

$$
\begin{aligned}
& \rho_{\varepsilon}(\theta)=\int_{0}^{\theta} \cos (\sqrt{\varepsilon} x) d x=\frac{1}{\sqrt{\varepsilon}} \sin (\sqrt{\varepsilon} x), \\
& \tau_{s}(l)=\frac{1}{2} e^{l}-\frac{1}{2} s e^{-l} .
\end{aligned}
$$

To be more explicit,

$$
\begin{array}{rlrl}
\rho_{1}(\theta) & =\sin (\theta), \quad \rho_{0}(\theta)=\theta, & \rho_{-1}(\theta) & =\sinh (\theta), \\
\tau_{1}(l) & =\sinh (l), \quad \tau_{0}(l)=\frac{1}{2} e^{l}, \quad \tau_{-1}(l) & =\cosh (l) .
\end{array}
$$

A simple calculation shows

$$
\begin{aligned}
\rho_{\varepsilon}^{\prime}(\theta) & :=\frac{\partial \rho_{\varepsilon}(\theta)}{\partial \theta}=\cos (\sqrt{\varepsilon} \theta), \\
\tau_{s}^{\prime}(l) & :=\frac{\partial \tau_{s}(l)}{\partial l}=\frac{1}{2} e^{l}+\frac{1}{2} s e^{-l} .
\end{aligned}
$$


Lemma 3.1 (The cosine laws and the sine laws) For a generalized hyperbolic triangle of type $\left(\varepsilon_{1}, \varepsilon_{2}, \varepsilon_{3}\right) \in\{-1,0,1\}^{3}$ with generalized angles $\theta_{1}, \theta_{2}, \theta_{3}$ and opposite generalized edge lengths $l_{1}, l_{2}, l_{3}$, for $\{i, j, k\}=\{1,2,3\}$, the following hold:

$$
\begin{aligned}
\tau_{\varepsilon_{j} \varepsilon_{k}}^{\prime}\left(l_{i}\right) & =\frac{\rho_{\varepsilon_{i}}^{\prime}\left(\theta_{i}\right)+\rho_{\varepsilon_{j}}^{\prime}\left(\theta_{j}\right) \rho_{\varepsilon_{k}}^{\prime}\left(\theta_{k}\right)}{\rho_{\varepsilon_{j}}\left(\theta_{j}\right) \rho_{\varepsilon_{k}}\left(\theta_{k}\right)}, \\
2 \rho_{\varepsilon_{i}}^{2}\left(\frac{\theta_{i}}{2}\right) & =\frac{\tau_{\varepsilon_{j} \varepsilon_{k}}^{\prime}\left(l_{i}\right)-\frac{1}{2} \varepsilon_{j} e^{l_{j}-l_{k}-\frac{1}{2} \varepsilon_{k} e^{l_{k}-l_{j}}}}{\tau_{\varepsilon_{k} \varepsilon_{i}}\left(l_{j}\right) \tau_{\varepsilon_{i} \varepsilon_{j}}\left(l_{k}\right)}, \\
\rho_{\varepsilon_{i}}^{\prime}\left(\theta_{i}\right) & =\frac{-\varepsilon_{i} \tau_{\varepsilon_{j} \varepsilon_{k}}^{\prime}\left(l_{i}\right)+\tau_{\varepsilon_{k} \varepsilon_{i}}^{\prime}\left(l_{j}\right) \tau_{\varepsilon_{i} \varepsilon_{j}}^{\prime}\left(l_{k}\right)}{\tau_{\varepsilon_{k} \varepsilon_{i}}\left(l_{j}\right) \tau_{\varepsilon_{i} \varepsilon_{j}}\left(l_{k}\right)}, \\
\frac{\rho_{\varepsilon_{i}}\left(\theta_{i}\right)}{\tau_{\varepsilon_{j} \varepsilon_{k}}\left(l_{i}\right)} & =\frac{\rho_{\varepsilon_{j}}\left(\theta_{j}\right)}{\tau_{\varepsilon_{k} \varepsilon_{i}}\left(l_{j}\right)} .
\end{aligned}
$$

Proof In Appendix A, the cosine law and the sine law for each type of the ten generalized hyperbolic triangles are listed. We check directly that all the formulas there fit the uniform formulas (3-3)-(3-6).

Remark 3.2 The identity (3-6) is called the sine law. The formula (3-4) is stronger than the formula (3-5). They are equivalent if $\varepsilon_{i}= \pm 1$. If $\varepsilon_{i}=0$, the formula (3-5) is trivial while the formula (3-4) expresses $\theta$ in term of lengths $l$.

For the six cases of generalized triangle without ideal vertices, there is a unified strategy to derive the cosine law (3-3) and (3-5) by using the hyperboloid model. This unified strategy is essentially given in [18, pages 74-82]. But this method does not work for the four cases of generalized triangle with ideal vertices.

The concepts of Gram matrix and angle Gram matrix of a generalized hyperbolic triangle are defined as follows. For a generalized hyperbolic triangle of type $\left(\varepsilon_{1}, \varepsilon_{2}, \varepsilon_{3}\right) \in$ $\{-1,0,1\}^{3}$ with generalized angles $\theta_{1}, \theta_{2}, \theta_{3}$ and opposite generalized edge lengths $l_{1}, l_{2}, l_{3}$, its Gram matrix is

$$
G_{l}:=-\left(\begin{array}{ccc}
\varepsilon_{1} & \tau_{\varepsilon_{1} \varepsilon_{2}}^{\prime}\left(l_{3}\right) & \tau_{\varepsilon_{3} \varepsilon_{1}}^{\prime}\left(l_{2}\right) \\
\tau_{\varepsilon_{1} \varepsilon_{2}}^{\prime}\left(l_{3}\right) & \varepsilon_{2} & \tau_{\varepsilon_{2} \varepsilon_{3}}^{\prime}\left(l_{1}\right) \\
\tau_{\varepsilon_{3} \varepsilon_{1}}^{\prime}\left(l_{2}\right) & \tau_{\varepsilon_{2} \varepsilon_{3}}^{\prime}\left(l_{1}\right) & \varepsilon_{3}
\end{array}\right)
$$

and its angle Gram matrix is

$$
G_{\theta}:=-\left(\begin{array}{ccc}
-1 & \rho_{\varepsilon_{3}}^{\prime}\left(\theta_{3}\right) & \rho_{\varepsilon_{2}}^{\prime}\left(\theta_{2}\right) \\
\rho_{\varepsilon_{3}}^{\prime}\left(\theta_{3}\right) & -1 & \rho_{\varepsilon_{1}}^{\prime}\left(\theta_{1}\right) \\
\rho_{\varepsilon_{2}}^{\prime}\left(\theta_{2}\right) & \rho_{\varepsilon_{1}}^{\prime}\left(\theta_{1}\right) & -1
\end{array}\right) .
$$


Lemma 3.3 The determinants of $G_{l}$ and $G_{\theta}$ satisfy the following:

$$
\begin{aligned}
& \operatorname{det} G_{l}=-\left(\tau_{\varepsilon_{k} \varepsilon_{i}}\left(l_{j}\right) \tau_{\varepsilon_{i} \varepsilon_{j}}\left(l_{k}\right) \rho_{\varepsilon_{i}}\left(\theta_{i}\right)\right)^{2} \\
& \operatorname{det} G_{\theta}=-\left(\rho_{\varepsilon_{j}}\left(\theta_{j}\right) \rho_{\varepsilon_{k}}\left(\theta_{k}\right) \tau_{\varepsilon_{j} \varepsilon_{k}}\left(l_{i}\right)\right)^{2}
\end{aligned}
$$

For a hyperbolic triangle, Lemma 3.3 is well-known. We will prove it for a generalized triangle in Appendix B.

By Lemma 3.3 and the sine laws (3-6), we see

$$
\begin{aligned}
M & :=\frac{1}{\sqrt{-\operatorname{det} G_{l}}}\left(\begin{array}{ccc}
\tau_{\varepsilon_{2} \varepsilon_{3}}\left(l_{1}\right) & 0 & 0 \\
0 & \tau_{\varepsilon_{3} \varepsilon_{1}}\left(l_{2}\right) & 0 \\
0 & 0 & \tau_{\varepsilon_{1} \varepsilon_{2}}\left(l_{3}\right)
\end{array}\right) \\
& =\frac{1}{\sqrt{-\operatorname{det} G_{\theta}}}\left(\begin{array}{ccc}
\rho_{\varepsilon_{1}}\left(\theta_{1}\right) & 0 & 0 \\
0 & \rho_{\varepsilon_{2}}\left(\theta_{2}\right) & 0 \\
0 & 0 & \rho_{\varepsilon_{3}}\left(\theta_{3}\right)
\end{array}\right) .
\end{aligned}
$$

Lemma 3.4 $M G_{l} M G_{\theta}=I$.

This lemma is a consequence of the cosine laws and the sine laws (3-3), (3-5) and (3-6). It is checked by direct calculation.

Let $y_{1}, y_{2}, y_{3}$ be three functions of variables $x_{1}, x_{2}, x_{3}$. Let $A=\left(\frac{\partial y_{i}}{\partial x_{j}}\right)_{3 \times 3}$ be the Jacobi matrix. Then the differentials $d y_{1}, d y_{2}, d y_{3}$ and $d x_{1}, d x_{2}, d x_{3}$ satisfy

$$
\left(\begin{array}{l}
d y_{1} \\
d y_{2} \\
d y_{3}
\end{array}\right)=A\left(\begin{array}{l}
d x_{1} \\
d x_{2} \\
d x_{3}
\end{array}\right) .
$$

Lemma 3.5 (The derivative cosine law) For a generalized hyperbolic triangle of type $\left(\varepsilon_{1}, \varepsilon_{2}, \varepsilon_{3}\right) \in\{-1,0,1\}^{3}$ with generalized angles $\theta_{1}, \theta_{2}, \theta_{3}$ and opposite generalized edge lengths $l_{1}, l_{2}, l_{3}$, the differentials of $l^{\prime} s$ and $\theta^{\prime} s$ satisfy the following relations:

$$
\begin{aligned}
& \left(\begin{array}{l}
d l_{1} \\
d l_{2} \\
d l_{3}
\end{array}\right)=M G_{l}\left(\begin{array}{l}
d \theta_{1} \\
d \theta_{2} \\
d \theta_{3}
\end{array}\right), \\
& \left(\begin{array}{l}
d \theta_{1} \\
d \theta_{2} \\
d \theta_{3}
\end{array}\right)=M G_{\theta}\left(\begin{array}{l}
d l_{1} \\
d l_{2} \\
d l_{3}
\end{array}\right) .
\end{aligned}
$$

We will prove Lemma 3.5 in Appendix B. 
In the rest of the section, we establish the existence of a generalized hyperbolic triangle of type $(\varepsilon, \varepsilon, \delta) \in\{-1,0,1\}^{3}$ with two given edge lengths $l_{1}, l_{2}$ and a generalized angle $\theta$ between them, where the generalized angles opposite to $l_{1}, l_{2}$ have type $\varepsilon$ and $\theta$ has type $\delta$. Recall $\stackrel{\circ}{I}_{\delta}$ and $J_{\varepsilon \delta}$ are in (1-1) and (1-2).

Fix type $(\varepsilon, \varepsilon, \delta) \in\{-1,0,1\}^{3}$. For a given $\theta \in \stackrel{\circ}{I}_{\delta}$, let's introduce the set $\mathcal{D}_{\varepsilon, \delta}(\theta)=$ $\left\{\left(l_{1}, l_{2}\right) \in\left(J_{\varepsilon \delta}\right)^{2} \mid\right.$ there exists a generalized hyperbolic triangle of type $(\varepsilon, \varepsilon, \delta)$ with two edge lengths $l_{1}, l_{2}$ so that the generalized angle between them is $\theta$ of type $\delta$ \}.

Lemma 3.6 (1) If $\varepsilon=1$ or 0 , then $\mathcal{D}_{\varepsilon, \delta}(\theta)=\left(J_{\varepsilon \delta}\right)^{2}$.

(2) If $(\varepsilon, \delta)=(-1,1)$, then

$$
\mathcal{D}_{\varepsilon, \delta}(\theta)=\left\{\left(l_{1}, l_{2}\right) \in \mathbb{R}_{>0}^{2} \mid \sinh l_{1} \sinh l_{2}-\cos \theta \cosh l_{1} \cosh l_{2}>1\right\} .
$$

(3) If $(\varepsilon, \delta)=(-1,0)$, then

$$
\mathcal{D}_{\varepsilon, \delta}(\theta)=\left\{\left(l_{1}, l_{2}\right) \in \mathbb{R}^{2} \mid \theta>e^{-l_{1}}+e^{-l_{2}}\right\} .
$$

(4) If $(\varepsilon, \delta)=(-1,-1)$, then

$$
\mathcal{D}_{\varepsilon, \delta}(\theta)=\left\{\left(l_{1}, l_{2}\right) \in \mathbb{R}_{>0}^{2} \mid \cosh \theta \sinh l_{1} \sinh l_{2}-\cosh l_{1} \cosh l_{2}>1\right\} .
$$

Proof We will construct a generalized hyperbolic triangle of type $(\varepsilon, \varepsilon, \delta)$ in each case as follows.

Denote by $\mathbb{H}^{2}$ the hyperbolic plane and by $\partial \mathbb{H}^{2}$ the ideal boundary of $\mathbb{H}^{2}$.

If $(\varepsilon, \varepsilon, \delta)=(1,1,1)$, choose a point $O \in \mathbb{H}^{2}$. Draw two geodesics rays $L_{1}, L_{2}$ starting from the point $O$ such that the angle between $L_{1}, L_{2}$ is $\theta \in(0, \pi)$. Let $P_{i}$ be the point on $L_{i}$ such that the length of the segment $O P_{i}$ is $l_{i}>0, i=1,2$. Then one obtains the hyperbolic triangle by joining $P_{1}, P_{2}$ by a geodesic segment.

If $(\varepsilon, \varepsilon, \delta)=(1,1,0)$, choose a point $O \in \partial \mathbb{H}^{2}$ and draw a horocycle centered at $O$. Let $H_{1}, H_{2}$ be two points on the horocycle such that the length of the horocyclic arc $H_{1} H_{2}$ is $\frac{\theta}{2}>0$. For $i=1,2$, draw a geodesic $L_{i}$ passing through $O$ and $H_{i}$. Let $P_{i}$ be the point on $L_{i}$ such that the length of the segment of $H_{i} P_{i}$ is $\left|l_{i}\right|$ and $H_{i}$ is between $O, P_{i}$ on $L_{i}$ if and only if $l_{i}>0$. Then one obtains the generalized hyperbolic triangle by joining $P_{1}, P_{2}$ by a geodesic segment.

If $(\varepsilon, \varepsilon, \delta)=(1,1,-1)$, draw a geodesic segment $G_{1} G_{2}$ of length $\theta>0$ with end points $G_{1}, G_{2}$. For $i=1,2$, let $L_{i}$ be a geodesic ray starting from $G_{i}$ perpendicular to $G_{1} G_{2}$ and $L_{1}, L_{2}$ are in the same half plane bounded by the geodesic containing $G_{1} G_{2}$. Let $P_{i}$ be the point on $L_{i}$ such that the length of the segment $G_{i} P_{i}$ is $l_{i}>0$, $i=1,2$. Then one obtains the generalized hyperbolic triangle by joining $P_{1}, P_{2}$ by a geodesic segment. 
If $(\varepsilon, \varepsilon, \delta)=(0,0,1)$, choose a point $O \in \mathbb{H}^{2}$. Draw two geodesics rays $L_{1}, L_{2}$ starting form the point $O$ such that the angle between $L_{1}, L_{2}$ is $\theta \in(0, \pi)$ and $L_{i}$ ends at point $P_{i} \in \partial \mathbb{H}^{2}$. Join $P_{1}, P_{2}$ by a geodesic. Let $H_{i}$ be the point on the geodesic containing $L_{i}$ such the length of $O H_{i}$ is $\left|l_{i}\right|$ and $H_{i}$ is between $O, P_{i}$ if and only if $l_{i}>0$. Draw a horocycle centered at $P_{i}$ passing through $H_{i}$. One obtains the generalized triangle $O P_{1} P_{2}$ decorated by two horocycles.

If $(\varepsilon, \varepsilon, \delta)=(0,0,0)$ or $(0,0,-1)$, the construction is similar to the case of $(0,0,1)$ above.

If $(\varepsilon, \varepsilon, \delta)=(-1,-1,1)$, choose a point $O \in \mathbb{H}^{2}$. Draw two geodesics rays $L_{1}, L_{2}$ starting form the point $O$ such that the angle between $L_{1}, L_{2}$ is $\theta \in(0, \pi)$. For $i=1,2$, let $P_{i}$ be the point on $L_{i}$ such that the length of the segment $O P_{i}$ is $l_{i}>0$. Let $M_{i}$ be the geodesic passing through $P_{i}$ perpendicular to $L_{i}$. There is a generalized hyperbolic triangle with prescribed lengths $l_{1}, l_{2}$ and angle $\theta$ if and only if the distance between $M_{1}, M_{2}$ is positive. The distance $l$ between $M_{1}, M_{2}$ can be calculated from the cosine law of generalized triangle of type $(-1,-1,1)$ :

$$
\cosh l=\sinh l_{1} \sinh l_{2}-\cos \theta \cosh l_{1} \cosh l_{2} .
$$

This shows that the condition in part (2) is equivalent to $l>0$.

If $(\varepsilon, \varepsilon, \delta)=(-1,-1,0)$, choose a point $O \in \partial \mathbb{H}^{2}$ and draw a horocycle centered at $O$. Let $H_{1}, H_{2}$ be the two points on the horocycle such that the length of the horocyclic arc $H_{1} H_{2}$ is $\frac{\theta}{2}>0$. For $i=1,2$, draw a geodesic $L_{i}$ passing through $O$ and $H_{i}$. Let $P_{i}$ be the point on $L_{i}$ such that the length of the segment $H_{i} P_{i}$ is $\left|l_{i}\right|$ and $H_{i}$ is between $O, P_{i}$ if and only if $l_{i}>0$. Let $M_{i}$ be the geodesic passing through $P_{i}$ perpendicular to $L_{i}$. There is a generalized hyperbolic triangle with prescribed lengths $l_{1}, l_{2}$ and generalized angle $\theta$ if and only if the distance $l$ between $M_{1}, M_{2}$ is positive. The distance $l$ between $M_{1}, M_{2}$ can be calculated from the cosine law of generalized triangle of type $(-1,-1,0)$ :

$$
\cosh l=\frac{1}{2} \theta^{2} e^{l_{1}+l_{2}}-\cosh \left(l_{1}-l_{2}\right) .
$$

Now, one sees $\frac{1}{2} \theta^{2} e^{l_{1}+l_{2}}-\cosh \left(l_{1}-l_{2}\right)>1$ if and only if $\theta>e^{-l_{1}}+e^{-l_{2}}$. This shows part (3) holds.

If $(\varepsilon, \varepsilon, \delta)=(-1,-1,-1)$, draw a geodesic segment $G_{1} G_{2}$ of length $\theta>0$ with end points $G_{1}, G_{2}$. For $i=1,2$, let $L_{i}$ be a geodesic half line starting from $G_{i}$ perpendicular to $G_{1} G_{2}$ and $L_{1}, L_{2}$ are in the same half plane bounded by the geodesic containing $G_{1} G_{2}$. Let $P_{i}$ be the point on $L_{i}$ such that the length of the segment $G_{i} P_{i}$ is $l_{i}>0, i=1,2$. Let $M_{i}$ be the geodesic passing through $P_{i}$ perpendicular to $L_{i}$. 
There is a right-angled hexagon with prescribed lengths $l_{1}, l_{2}, \theta$ if and only if the distance $l$ between $M_{1}, M_{2}$ is positive. The distance $l$ between $M_{1}, M_{2}$ can be calculated from the cosine law of a right-angled hexagon:

$$
\cosh l=\cosh \theta \sinh l_{1} \sinh l_{2}-\cosh l_{1} \cosh l_{2} .
$$

This shows that the condition in part (4) is equivalent to $l>0$.

\section{A proof of Theorem 1.4}

We give a proof of the generalized circle packing theorem (Theorem 1.4) in this section. Recall that $(\Sigma, T)$ is a closed triangulated surface with $V, E, F$ the sets all vertices, edges and triangles in $T$. Given a type $(\varepsilon, \varepsilon, \delta) \in\{-1,0,1\}^{3}$ and $\Phi: E \rightarrow I_{\delta}$, for each $r \in \mathcal{N}_{\varepsilon, \delta}(\Phi)$, a generalized $(\varepsilon, \varepsilon, \delta)$ circle packing on $(\Sigma, T)$ is based on the following local construction.

As in Figure 12, consider a topological triangle with vertices $v_{i}, v_{j}, v_{k}$. One can construct a generalized hyperbolic triangle $\Delta v_{i} v_{j} q_{k}$ of type $(\varepsilon, \varepsilon, \delta)$ such that the edges $v_{i} q_{k}, v_{j} q_{k}$ have lengths $r\left(v_{i}\right):=r_{i}, r\left(v_{j}\right):=r_{j}$ respectively and the generalized angle at $q_{k}$ is $\Phi\left(v_{i} v_{j}\right)=: \phi_{k}$. Let $l\left(v_{i} v_{j}\right)=: l_{k}$ be the length of edge $v_{i} v_{j}$ in the generalized hyperbolic triangle $\triangle v_{i} v_{j} q_{k}$ which is a function of $r_{i}, r_{j}$. Similarly, one obtains the edge lengths $l\left(v_{j} v_{k}\right)=: l_{i}, l\left(v_{k} v_{i}\right):=l_{j}$. Let $\theta_{i}$ be the generalized angle of $\Delta v_{i} v_{j} v_{k}$ at the vertex $v_{i}$. With fixed $\left(\phi_{i}, \phi_{j}, \phi_{k}\right)$, we consider $\theta_{i}$ as a function of $\left(r_{i}, r_{j}, r_{k}\right)$.

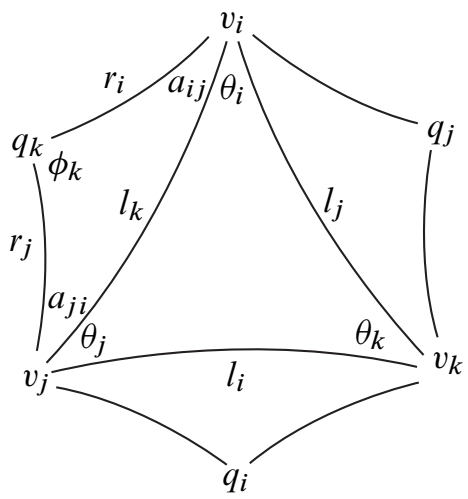

Figure 12: Data for generalized circle packing

Let's recall the definition of $\mathcal{M}_{\varepsilon, \delta}\left(\Phi\left(v_{i} v_{j}\right), \Phi\left(v_{j} v_{k}\right), \Phi\left(v_{k} v_{i}\right)\right)$ in Section 1.4 and definition of $\mathcal{D}_{\varepsilon, \delta}(\theta)$ in Section 3. 
Lemma 4.1 When $\varepsilon=0$ or -1 , we have

$$
\mathcal{M}_{\varepsilon, \delta}\left(\phi_{1}, \phi_{2}, \phi_{3}\right)=\left\{\left(r_{1}, r_{2}, r_{3}\right) \in\left(J_{\varepsilon \delta}\right)^{3} \mid\left(r_{i}, r_{j}\right) \in \mathcal{D}_{\varepsilon, \delta}\left(\phi_{k}\right),\{i, j, k\}=\{1,2,3\}\right\} .
$$

Proof Given $\phi_{k} \in \stackrel{\circ}{I}_{\delta}$, by Lemma 3.6, for $r_{i}, r_{j} \in \mathcal{D}_{\varepsilon, \delta}\left(\phi_{k}\right)$, there is generalized hyperbolic triangle $\triangle v_{i} v_{j} q_{k}$ of type $(\varepsilon, \varepsilon, \delta)$ with edge lengths $r_{i}, r_{j}$ and angle $\phi_{k}$ of type $\delta$ between the two edges. We obtain the edge length $l_{k}=l\left(v_{i} v_{j}\right)$. There is a case which is not contained in Lemma 3.6: $\delta=1, \phi_{k}=\pi$. It is easier since we have $l_{k}=r_{i}+r_{j}$.

For $\varepsilon=0$, we get $l_{i}, l_{j}, l_{k} \in \mathbb{R}$. Thus there exists a decorated ideal triangle with three edge lengths $l_{i}, l_{j}, l_{k}$. For $\varepsilon=-1$, the inequality defining $\mathcal{D}_{-1, \delta}\left(\phi_{k}\right)$ guarantees that $l_{i}, l_{j}, l_{k}>0$. Thus there is a right-angled hexagon with three edge lengths $l_{i}, l_{j}, l_{k} . \square$

\subsection{A proof of Theorem 1.4 for $\varepsilon=0$}

We assume that indices $i, j, k$ are distinct in this section. First by Lemma 3.6 and Lemma 4.1 , we have $\mathcal{M}_{0, \delta}\left(\phi_{i}, \phi_{j}, \phi_{k}\right)=\mathbb{R}^{3}$. Therefore $\mathcal{N}_{0, \delta}(\Phi)=\mathbb{R}^{V}$.

The case $\varepsilon=0$ is very simple. Indeed, for a fixed $\Phi: E \rightarrow I_{\delta}$, there exists a map $C: V \rightarrow \mathbb{R}_{>0}$ so that for all $r \in \mathcal{N}_{0, \delta}(\Phi)=\mathbb{R}^{V}$,

$$
\widetilde{K}(r)(v)=C(v) e^{-r(v)},
$$

ie, the discrete curvature $\tilde{K}(r)$ is uniformly proportional to $e^{-r(v)}$. Thus, one sees easily that the generalized circle packing metric $r \in \mathbb{R}^{V}$ is determined by its generalized discrete curvature $\tilde{K}: V \rightarrow \mathbb{R}$ and $\{\tilde{K}(v) \mid v \in V\}=\mathbb{R}_{>0}^{V}$.

Indeed, in Figure 12, the generalized triangle $\triangle v_{i} v_{j} q_{k}$ has type $(0,0, \delta)$, edge lengths $r_{i}, r_{j}, l_{k}$ and inner angle $\phi_{k}$ opposite to $l_{k}$. By the cosine law (3-4), we have

$$
2 \rho_{\delta}^{2}\left(\frac{\phi_{k}}{2}\right)=\frac{\tau_{0}^{\prime}\left(l_{k}\right)}{\tau_{0}\left(r_{i}\right) \tau_{0}\left(r_{j}\right)}=2 e^{l_{k}-r_{i}-r_{j}}
$$

Thus

$$
e^{l_{k}}=\rho_{\delta}^{2}\left(\frac{\phi_{k}}{2}\right) e^{r_{i}+r_{j}}
$$

By the cosine law (3-4) for the decorated ideal triangle $\Delta v_{i} v_{j} v_{k}$ (or type $(0,0,0)$ generalized triangle) we have

$$
\frac{\theta_{k}^{2}}{4}=e^{l_{k}-l_{i}-l_{j}}
$$


Combining (4-2) and (4-3) we obtain

$$
\frac{\theta_{k}^{2}}{4}=e^{l_{k}-l_{i}-l_{j}}=\frac{\rho_{\delta}^{2}\left(\frac{\phi_{k}}{2}\right) e^{r_{i}+r_{j}}}{\rho_{\delta}^{2}\left(\frac{\phi_{i}}{2}\right) e^{r_{j}+r_{k}} \rho_{\delta}^{2}\left(\frac{\phi_{j}}{2}\right) e^{r_{k}+r_{i}}}=\frac{\rho_{\delta}^{2}\left(\frac{\phi_{k}}{2}\right)}{\rho_{\delta}^{2}\left(\frac{\phi_{i}}{2}\right) \rho_{\delta}^{2}\left(\frac{\phi_{j}}{2}\right)} e^{-2 r_{k}},
$$

hence

$$
\theta_{k}=\frac{2 \rho_{\delta}\left(\frac{\phi_{k}}{2}\right)}{\rho_{\delta}\left(\frac{\phi_{i}}{2}\right) \rho_{\delta}\left(\frac{\phi_{j}}{2}\right)} e^{-r_{k}}
$$

Summing up (4-4) for all triangle having $v_{k}$ as a vertex, we obtain (4-1) where $C(v)$ depends explicitly on $\delta$.

\subsection{Rigidity of circle packing for $\varepsilon=-1$ or $(\varepsilon, \delta)=(1,1)$}

In this subsection we prove Theorem 1.4 for the case $\varepsilon=-1$ and give a new proof of Thurston's rigidity Theorem 1.3.

In the variational framework, the natural parameter is $u=\left(u_{1}, u_{2}, u_{3}\right)$ where

$$
u_{i}=-\int_{r_{i}}^{\infty} \frac{1}{\tau_{\varepsilon \delta}(t)} d t
$$

for $\varepsilon= \pm 1$. Note that by the definition of $\tau_{s}, \partial u_{i} / \partial r_{i}>0$.

Lemma 4.2 Fix $\phi=\left(\phi_{1}, \phi_{2}, \phi_{3}\right) \in I_{\delta}^{3}$, the set $u\left(\mathcal{M}_{\varepsilon, \delta}\left(\phi_{1}, \phi_{2}, \phi_{3}\right)\right)$ is an open convex polyhedron in $\mathbb{R}^{3}$ in the following cases:

(i) $\varepsilon=-1$, or

(ii) $(\varepsilon, \delta)=(1,1)$ and $\phi_{i} \in\left[\frac{\pi}{2}, \pi\right], i=1,2,3$.

Proof (i) When $\varepsilon=-1$, we have figured out the set $\mathcal{M}_{\varepsilon, \delta}\left(\phi_{1}, \phi_{2}, \phi_{3}\right)$ in Lemma 4.1.

If the type of $\phi_{k}$ is $\delta=1$, by the proof of Lemma 3.6 (2), we need $l_{k}>0$ which is the same as

$$
\cos \phi_{k}=\frac{-\cosh l_{k}+\sinh r_{i} \sinh r_{j}}{\cosh r_{i} \cosh r_{j}}<\frac{-1+\sinh r_{i} \sinh r_{j}}{\cosh r_{i} \cosh r_{j}} .
$$

In this case $u_{i}=-\int_{r_{i}}^{\infty} 1 / \cosh t d t=2 \arctan e^{r_{i}}-\pi \in\left(-\frac{\pi}{2}, 0\right)$. Then

$$
\sinh r_{i}=-\frac{1}{\tan u_{i}}, \quad \cosh r_{i}=-\frac{1}{\sin u_{i}} .
$$


By substituting (4-7) into (4-6), we obtain $\cos \phi_{k}<\cos \left(u_{i}+u_{j}\right)$. Since $\phi_{k} \in$ $(0, \pi],-u_{i}-u_{j} \in(0, \pi)$, we see that $l_{k}>0$ if and only if $u_{i}+u_{j}>-\phi_{k}$. Then $u\left(\mathcal{M}_{\varepsilon, \delta}\left(\phi_{1}, \phi_{2}, \phi_{3}\right)\right)=\left\{\left(u_{1}, u_{2}, u_{3}\right) \in \mathbb{R}_{<0}^{3} \mid u_{i}+u_{j}>-\phi_{k},\{i, j, k\}=\{1,2,3\}\right\}$. The last description shows that $u\left(\mathcal{M}_{\varepsilon, \delta}\left(\phi_{1}, \phi_{2}, \phi_{3}\right)\right)$ is a convex polyhedron.

If the type of $\phi_{k}$ is $\delta=0$, we need $l_{k}>0$ which is equivalent to

$$
\frac{\phi_{k}^{2}}{2}=\frac{\cosh l_{k}+\cosh \left(r_{i}-r_{j}\right)}{e^{r_{i}+r_{j}}}>\frac{1+\cosh \left(r_{i}-r_{j}\right)}{e^{r_{i}+r_{j}}}=\frac{1}{2}\left(e^{-r_{i}}+e^{-r_{j}}\right)^{2} .
$$

Since in this case $u_{i}=-\int_{r_{i}}^{\infty} 1 / e^{t} d t=-e^{-r_{i}}<0$, we see that $l_{k}>0$ is the same as having $u_{i}+u_{j}>-\phi_{k}$. Thus $u\left(\mathcal{M}_{\varepsilon, \delta}\left(\phi_{1}, \phi_{2}, \phi_{3}\right)\right)=\left\{\left(u_{1}, u_{2}, u_{3}\right) \in \mathbb{R}_{<0}^{3} \mid l_{i}>0\right.$ for all $i\}=\left\{\left(u_{1}, u_{2}, u_{3}\right) \in \mathbb{R}_{<0}^{3} \mid u_{i}+u_{j}>-\phi_{k},\{i, j, k\}=\{1,2,3\}\right\}$. The last description shows that $u\left(\mathcal{M}_{\varepsilon, \delta}\left(\phi_{1}, \phi_{2}, \phi_{3}\right)\right)$ is a convex polyhedron.

If the type of $\phi_{k}$ is $\delta=-1$, we need $l_{k}>0$ which is the same as

$$
\begin{aligned}
\cosh \phi_{k} & =\frac{\cosh l_{k}+\cosh r_{i} \cosh r_{j}}{\sinh r_{i} \sinh r_{j}} \\
& >\frac{1+\cosh r_{i} \cosh r_{j}}{\sinh r_{i} \sinh r_{j}} \\
& =\frac{1}{2}\left(\tanh \frac{r_{i}}{2} \tanh \frac{r_{j}}{2}+\frac{1}{\tanh \frac{r_{i}}{2} \tanh \frac{r_{j}}{2}}\right) .
\end{aligned}
$$

Hence $l_{k}>0$ if and only if

$$
e^{-\phi_{k}}<\tanh \frac{r_{i}}{2} \tanh \frac{r_{j}}{2}
$$

It follows that $l_{k}>0$ is the same as

$$
\ln \tanh \frac{r_{i}}{2}+\ln \tanh \frac{r_{j}}{2}>-\phi_{k} .
$$

Since in this case $u_{i}=-\int_{r_{i}}^{\infty} 1 / \sinh t d t=\ln \tanh \frac{r_{i}}{2}<0$, we see that $l_{k}>0$ if and only if $u_{i}+u_{j}>-\phi_{k}$. Thus $u\left(\mathcal{M}_{\varepsilon, \delta}\left(\phi_{1}, \phi_{2}, \phi_{3}\right)\right)=\left\{\left(u_{1}, u_{2}, u_{3}\right) \in \mathbb{R}_{<0}^{3} \mid u_{i}+u_{j}>-\phi_{k}\right.$, $\{i, j, k\}=\{1,2,3\}\}$. The last description shows that $u\left(\mathcal{M}_{\varepsilon, \delta}\left(\phi_{1}, \phi_{2}, \phi_{3}\right)\right)$ is a convex polyhedron.

(ii) When $\varepsilon=1, \delta=1$ and $\phi_{k} \in\left[\frac{\pi}{2}, \pi\right]$, Thurston observed $\mathcal{M}_{1,1}\left(\phi_{1}, \phi_{2}, \phi_{3}\right)=\mathbb{R}_{>0}^{3}$. Indeed, $\phi_{i} \in\left[\frac{\pi}{2}, \pi\right]$ implies $l_{i}>r_{j}$. Similarly, $l_{j}>r_{i}$. See Figure 12. Hence $l_{i}+l_{j}>$ $r_{j}+r_{i}>l_{k}$. This shows that $l_{1}, l_{2}, l_{3}$ are the lengths of a hyperbolic triangle. In this case $u_{i}=-\int_{r_{i}}^{\infty} 1 / \sinh t d t=\ln \tanh \frac{r_{i}}{2}<0$. Thus $u\left(\mathcal{M}_{1,1}\left(\phi_{1}, \phi_{2}, \phi_{3}\right)\right)=\mathbb{R}_{<0}^{3}$.

Lemma 4.3 For $\varepsilon= \pm 1$ and fixed $\left(\phi_{1}, \phi_{2}, \phi_{3}\right) \in I_{\delta}^{3}$, the Jacobi matrix $A$ of the function $\left(\theta_{1}, \theta_{2}, \theta_{3}\right)=\left(\theta_{1}(u), \theta_{2}(u), \theta_{3}(u)\right): u\left(\mathcal{M}_{\varepsilon, \delta}\left(\phi_{1}, \phi_{2}, \phi_{3}\right)\right) \rightarrow \mathbb{R}^{3}$ is symmetric. 
Proof Consider the $(\varepsilon, \varepsilon, \delta)$-triangle $\triangle v_{i} v_{j} q_{k}$ in Figure 12 with edge lengths $r_{i}, r_{j}$, $l_{k}$ and opposite generalized angles $a_{j i}, a_{i j}, \phi_{k}$. By applying the derivative cosine law (3-10) to $\triangle v_{i} v_{j} q_{k}$, the third row of the matrix in (3-10) is

$$
d \phi_{k}=\frac{-\rho_{\delta}\left(\phi_{k}\right)}{\sqrt{-\operatorname{det} G_{\theta}}}\left(\rho_{\varepsilon}^{\prime}\left(a_{j i}\right) d r_{i}+\rho_{\varepsilon}^{\prime}\left(a_{i j}\right) d r_{j}-d l_{k}\right) .
$$

Since $\phi_{k}$ is fixed, then $d \phi_{k}=0$. We have $d l_{k}=\rho_{\varepsilon}^{\prime}\left(a_{j i}\right) d r_{i}+\rho_{\varepsilon}^{\prime}\left(a_{i j}\right) d r_{j}$. For $\varepsilon= \pm 1$, we have $u_{i}=-\int_{r_{i}}^{\infty} 1 / \tau_{\varepsilon \delta}(t) d t$. Then $d r_{i}=\tau_{\varepsilon \delta}\left(r_{i}\right) d u_{i}$. Thus

$$
\left(\begin{array}{l}
d l_{1} \\
d l_{2} \\
d l_{3}
\end{array}\right)=\left(\begin{array}{ccc}
0 & \rho_{\varepsilon}^{\prime}\left(a_{23}\right) & \rho_{\varepsilon}^{\prime}\left(a_{32}\right) \\
\rho_{\varepsilon}^{\prime}\left(a_{13}\right) & 0 & \rho_{\varepsilon}^{\prime}\left(a_{31}\right) \\
\rho_{\varepsilon}^{\prime}\left(a_{12}\right) & \rho_{\varepsilon}^{\prime}\left(a_{21}\right) & 0
\end{array}\right)\left(\begin{array}{l}
d r_{1} \\
d r_{2} \\
d r_{3}
\end{array}\right)
$$

$$
=\left(\begin{array}{ccc}
0 & \rho_{\varepsilon}^{\prime}\left(a_{23}\right) & \rho_{\varepsilon}^{\prime}\left(a_{32}\right) \\
\rho_{\varepsilon}^{\prime}\left(a_{13}\right) & 0 & \rho_{\varepsilon}^{\prime}\left(a_{31}\right) \\
\rho_{\varepsilon}^{\prime}\left(a_{12}\right) & \rho_{\varepsilon}^{\prime}\left(a_{21}\right) & 0
\end{array}\right)\left(\begin{array}{ccc}
\tau_{\varepsilon \delta}\left(r_{1}\right) & 0 & 0 \\
0 & \tau_{\varepsilon \delta}\left(r_{2}\right) & 0 \\
0 & 0 & \tau_{\varepsilon \delta}\left(r_{3}\right)
\end{array}\right)\left(\begin{array}{l}
d u_{1} \\
d u_{2} \\
d u_{3}
\end{array}\right) .
$$

For the $(\varepsilon, \varepsilon, \varepsilon)$-triangle $\Delta v_{1} v_{2} v_{3}$ with angles $\theta_{1}, \theta_{2}, \theta_{3}$ and edge lengths $l_{1}, l_{2}, l_{3}$, by the derivative cosine law (3-9) and (4-8), we have

$$
\begin{aligned}
\left(\begin{array}{l}
d \theta_{1} \\
d \theta_{2} \\
d \theta_{3}
\end{array}\right)= & \frac{-1}{\sqrt{-\operatorname{det} G_{l}}}\left(\begin{array}{ccc}
\tau_{1}\left(l_{1}\right) & 0 & 0 \\
0 & \tau_{1}\left(l_{2}\right) & 0 \\
0 & 0 & \tau_{1}\left(l_{3}\right)
\end{array}\right)\left(\begin{array}{ccc}
-1 & \rho_{\varepsilon}^{\prime}\left(\theta_{3}\right) & \rho_{\varepsilon}^{\prime}\left(\theta_{2}\right) \\
\rho_{\varepsilon}^{\prime}\left(\theta_{3}\right) & -1 & \rho_{\varepsilon}^{\prime}\left(\theta_{1}\right) \\
\rho_{\varepsilon}^{\prime}\left(\theta_{2}\right) & \rho_{\varepsilon}^{\prime}\left(\theta_{1}\right) & -1
\end{array}\right) \\
& \left(\begin{array}{cccc}
0 & \rho_{\varepsilon}^{\prime}\left(a_{23}\right) & \rho_{\varepsilon}^{\prime}\left(a_{32}\right) \\
\rho_{\varepsilon}^{\prime}\left(a_{13}\right) & 0 & \rho_{\varepsilon}^{\prime}\left(a_{31}\right) \\
\rho_{\varepsilon}^{\prime}\left(a_{12}\right) & \rho_{\varepsilon}^{\prime}\left(a_{21}\right) & 0
\end{array}\right)\left(\begin{array}{ccc}
\tau_{\varepsilon \delta}\left(r_{1}\right) & 0 & 0 \\
0 & \tau_{\varepsilon \delta}\left(r_{2}\right) & 0 \\
0 & 0 & \tau_{\varepsilon \delta}\left(r_{3}\right)
\end{array}\right)\left(\begin{array}{l}
d u_{1} \\
d u_{2} \\
d u_{3}
\end{array}\right) \\
= & : \frac{-1}{\sqrt{-\operatorname{det} G_{l}}} N\left(\begin{array}{c}
d u_{1} \\
d u_{2} \\
d u_{3}
\end{array}\right) .
\end{aligned}
$$

To show the Jacobi matrix $A$ of $\left(\theta_{1}(u), \theta_{2}(u), \theta_{3}(u)\right)$ is symmetric, we only need to check that $N=\left(N_{i j}\right)$ is symmetric. In fact

$$
N_{i j}=\tau_{1}\left(l_{i}\right) \tau_{\varepsilon \delta}\left(r_{j}\right)\left(-\rho_{\varepsilon}^{\prime}\left(a_{j k}\right)+\rho_{\varepsilon}^{\prime}\left(\theta_{j}\right) \rho_{\varepsilon}^{\prime}\left(a_{j i}\right)\right) .
$$

By the cosine law (3-5),

$$
\begin{aligned}
N_{i j}=\tau_{1}\left(l_{i}\right) \tau_{\varepsilon \delta}\left(r_{j}\right)\left(-\frac{\varepsilon \tau_{\varepsilon \delta}^{\prime}\left(r_{k}\right)}{\tau_{\varepsilon \delta}\left(r_{j}\right) \tau_{1}\left(l_{i}\right)}\right. & \\
& \left.+\frac{\varepsilon \tau_{1}^{\prime}\left(l_{j}\right)+\tau_{1}^{\prime}\left(l_{i}\right) \tau_{1}^{\prime}\left(l_{k}\right)}{\tau_{1}\left(l_{i}\right) \tau_{1}\left(l_{k}\right)} \frac{\varepsilon \tau_{\varepsilon \delta}^{\prime}\left(r_{i}\right)+\tau_{\varepsilon \delta}^{\prime}\left(r_{j}\right) \tau_{1}^{\prime}\left(l_{k}\right)}{\tau_{\varepsilon \delta}\left(r_{j}\right) \tau_{1}\left(l_{k}\right)}\right)
\end{aligned}
$$




$$
\begin{aligned}
=\frac{\varepsilon \tau_{1}^{\prime}\left(l_{k}\right)\left(-\tau_{1}^{2}\left(l_{k}\right)+\tau_{\varepsilon \delta}^{\prime}\left(r_{j}\right) \tau_{1}^{\prime}\left(l_{j}\right)+\tau_{\varepsilon \delta}^{\prime}\left(r_{i}\right) \tau_{1}^{\prime}\left(l_{i}\right)\right)}{\tau_{1}^{2}\left(l_{k}\right)} \\
\quad+\frac{\left(\tau_{1}^{\prime 2}\left(l_{k}\right)-\tau_{1}^{2}\left(l_{k}\right)\right) \tau_{\varepsilon \delta}^{\prime}\left(r_{j}\right) \tau_{1}^{\prime}\left(l_{i}\right)+\varepsilon^{2} \tau_{\varepsilon \delta}^{\prime}\left(r_{i}\right) \tau_{1}^{\prime}\left(l_{j}\right)}{\tau_{1}^{2}\left(l_{k}\right)} .
\end{aligned}
$$

Since $\varepsilon= \pm 1, \tau_{1}\left(l_{k}\right)=\frac{1}{2} e^{l_{k}}-\frac{1}{2} e^{-l_{k}}=\sinh l_{k}$, we have $\tau_{1}^{\prime 2}\left(l_{k}\right)-\tau_{1}^{2}\left(l_{k}\right)=1=\varepsilon^{2}$. Therefore $N_{i j}$ is symmetric in the index $i, j$.

Lemma 4.4 Given $\left(\phi_{1}, \phi_{2}, \phi_{3}\right) \in I_{\delta}^{3}$, the Jacobi matrix $A$ of

$$
\left(\theta_{1}, \theta_{2}, \theta_{3}\right)=\left(\theta_{1}(u), \theta_{2}(u), \theta_{3}(u)\right): u\left(\mathcal{M}_{\varepsilon, \delta}\left(\phi_{1}, \phi_{2}, \phi_{3}\right)\right) \rightarrow \mathbb{R}^{3}
$$

is negative definite in the following cases:

(i) $\varepsilon=-1$ or

(ii) (Colin de Verdiére [4], Chow-Luo [3]) $(\varepsilon, \delta)=(1,1)$ and $\phi_{i} \in\left[\frac{\pi}{2}, \pi\right], i=$ $1,2,3$.

We remark that the case (ii) for $\phi_{i}=\pi$ was first proved by Colin de Verdiére [4], and was proved for $\phi_{i} \in\left[\frac{\pi}{2}, \pi\right]$ in [3] using the Maple program. Our proof of (ii) is new.

Proof In the proof of Lemma 4.3, the Jacobi matrix $A=\left(-1 / \sqrt{-\operatorname{det} G_{l}}\right) N$. Hence it is sufficient to show $N$ is positive definite. First $\operatorname{det} N>0$. Indeed $N$ is a product of four matrixes. The first one and forth one are diagonal matrixes with positive determinant. By Lemma 3.3, the determinant of the second matrix (negative of an angle Gram matrix) is positive. The determinant of the third matrix is $\rho_{\varepsilon}^{\prime}\left(a_{12}\right) \rho_{\varepsilon}^{\prime}\left(a_{23}\right) \rho_{\varepsilon}^{\prime}\left(a_{31}\right)+\rho_{\varepsilon}^{\prime}\left(a_{32}\right) \rho_{\varepsilon}^{\prime}\left(a_{21}\right) \rho_{\varepsilon}^{\prime}\left(a_{13}\right)>0$. If $\varepsilon=-1$, the above inequality is obvious. If $\varepsilon=1$, for $\{i, j, k\}=\{1,2,3\}, \phi_{k}, a_{i j}, a_{j i}$ are inner angles of a hyperbolic triangle as in Figure 12. By the assumption $\phi_{k} \in\left[\frac{\pi}{2}, \pi\right]$, we must have $a_{i j}\left(0, \frac{\pi}{2}\right)$. Therefore $\rho_{1}^{\prime}\left(a_{i j}\right)=\cos \left(a_{i j}\right)>0$. Thus the above inequality holds.

Since the set $u\left(\mathcal{M}_{\varepsilon, \delta}\left(\phi_{1}, \phi_{2}, \phi_{3}\right)\right)$ is connected and the $\operatorname{det} N \neq 0$, to show $N$ is positive definite, we only need to check $N$ is positive definite at one point in $u\left(\mathcal{M}_{\varepsilon, \delta}\left(\phi_{1}, \phi_{2}, \phi_{3}\right)\right)$. We choose the point such that $r_{1}=r_{2}=r_{3}=r$.

Now in Figure 12 and Figure 13, in the $(\varepsilon, \varepsilon, \delta)$-triangle $\Delta v_{i} v_{j} q_{k}$ with edge lengths $r, r, l_{k}$ and opposite generalized angles $a_{i j}=a_{j i}, \phi_{k}$, let $h$ be the geodesic length realizing the distance between the edge $v_{i} v_{j}$ and the generalized vertex $q_{k}$. In the $(\varepsilon, 1, \delta)$-triangle $\Delta v_{i} R q_{k}$, where $R q_{k}$ is perpendicular to $v_{i} v_{j}$, by the cosine law (3-5), we have

$$
\cos \frac{\pi}{2}=\frac{-\tau_{\varepsilon \delta}^{\prime}(r)+\tau_{\delta}^{\prime}(h) \tau_{\varepsilon}^{\prime}\left(\frac{l_{k}}{2}\right)}{\tau_{\delta}(h) \tau_{\varepsilon}\left(\frac{l_{k}}{2}\right)} .
$$




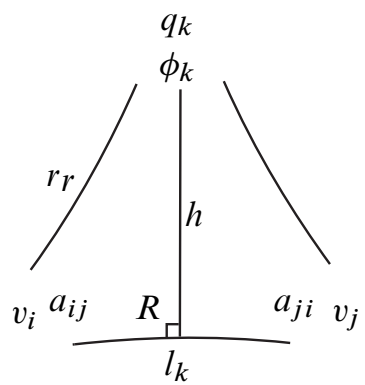

Figure 13: Data for generalized circle packing

Thus $\tau_{\delta}^{\prime}(h)=\tau_{\varepsilon \delta}^{\prime}(r) / \tau_{\varepsilon}^{\prime}\left(\frac{l_{k}}{2}\right)$. Also in $\Delta v_{i} R q_{k}$, by the cosine law (3-5), we have

$$
\rho_{\varepsilon}^{\prime}\left(a_{i j}\right)=\frac{-\varepsilon \tau_{\delta}^{\prime}(h)+\tau_{\varepsilon}^{\prime}\left(\frac{l_{k}}{2}\right) \tau_{\varepsilon \delta}^{\prime}(r)}{\tau_{\varepsilon}\left(\frac{l_{k}}{2}\right) \tau_{\varepsilon \delta}(r)}=\frac{\tau_{\varepsilon \delta}^{\prime}(r)\left(-\varepsilon+\tau_{\varepsilon}^{\prime 2}\left(\frac{l_{k}}{2}\right)\right)}{\tau_{\varepsilon}^{\prime}\left(\frac{l_{k}}{2}\right) \tau\left(\frac{l_{k}}{2}\right) \tau_{\varepsilon \delta}(r)} .
$$

Since

$$
-\varepsilon+\tau_{\varepsilon}^{\prime 2}(l)=-\varepsilon+\left(\frac{1}{2} e^{l}+\frac{1}{2} \varepsilon e^{-l}\right)^{2}=\left(\frac{1}{2} e^{l}-\frac{1}{2} \varepsilon e^{-l}\right)^{2}=\tau_{\varepsilon}^{2}(l),
$$

we have

$$
\rho_{\varepsilon}^{\prime}\left(a_{i j}\right)=\frac{\tau_{\varepsilon \delta}^{\prime}(r) \tau_{\varepsilon}\left(\frac{l_{k}}{2}\right)}{\tau_{\varepsilon \delta}(r) \tau_{\varepsilon}^{\prime}\left(\frac{l_{k}}{2}\right)}
$$

By substituting (4-9) into $N$, let

$$
N_{1}=\left(\begin{array}{ccc}
-1 & \rho_{\varepsilon}^{\prime}\left(\theta_{3}\right) & \rho_{\varepsilon}^{\prime}\left(\theta_{2}\right) \\
\rho_{\varepsilon}^{\prime}\left(\theta_{3}\right) & -1 & \rho_{\varepsilon}^{\prime}\left(\theta_{1}\right) \\
\rho_{\varepsilon}^{\prime}\left(\theta_{2}\right) & \rho_{\varepsilon}^{\prime}\left(\theta_{1}\right) & -1
\end{array}\right)\left(\begin{array}{ccc}
0 & \tau_{\varepsilon}\left(\frac{l_{1}}{2}\right) / \tau_{\varepsilon}^{\prime}\left(\frac{l_{1}}{2}\right) & \tau_{\varepsilon}\left(\frac{l_{1}}{2}\right) / \tau_{\varepsilon}^{\prime}\left(\frac{l_{1}}{2}\right) \\
\tau_{\varepsilon}\left(\frac{l_{2}}{2}\right) / \tau_{\varepsilon}^{\prime}\left(\frac{l_{2}}{2}\right) & 0 & \tau_{\varepsilon}\left(\frac{l_{2}}{2}\right) / \tau_{\varepsilon}^{\prime}\left(\frac{l_{2}}{2}\right) \\
\tau_{\varepsilon}\left(\frac{l_{3}}{2}\right) / \tau_{\varepsilon}^{\prime}\left(\frac{l_{3}}{2}\right) & \tau_{\varepsilon}\left(\frac{l_{3}}{2}\right) / \tau_{\varepsilon}^{\prime}\left(\frac{l_{3}}{2}\right) & 0
\end{array}\right) .
$$

Then $N, N_{1}$ differ by left and right multiplication by diagonal matrices of positive diagonal entries. We will show that the determinants of the $1 \times 1$ and $2 \times 2$ principal submatrices of $N_{1}$ are positive. This implies that the determinants of the $1 \times 1$ and $2 \times 2$ principal submatrices of $N$ are positive. Thus $N$ is positive definite.

Indeed, the determinant of the $1 \times 1$ principal submatrix of $N_{1}$ is

$$
\rho_{\varepsilon}^{\prime}\left(\theta_{3}\right) \frac{\tau_{\varepsilon}\left(\frac{l_{2}}{2}\right)}{\tau_{\varepsilon}^{\prime}\left(\frac{l_{2}}{2}\right)}+\rho_{\varepsilon}^{\prime}\left(\theta_{2}\right) \frac{\tau_{\varepsilon}\left(\frac{l_{3}}{2}\right)}{\tau_{\varepsilon}^{\prime}\left(\frac{l_{3}}{2}\right)}
$$


The determinant of the $2 \times 2$ principal matrix of $N_{1}$ is

$$
\begin{aligned}
\left(\rho_{\varepsilon}^{\prime 2}\left(\theta_{3}\right)-1\right) \frac{\tau_{\varepsilon}\left(\frac{l_{1}}{2}\right)}{\tau_{\varepsilon}^{\prime}\left(\frac{l_{1}}{2}\right)} \frac{\tau_{\varepsilon}\left(\frac{l_{2}}{2}\right)}{\tau_{\varepsilon}^{\prime}\left(\frac{l_{2}}{2}\right)} & +\left(\rho_{\varepsilon}^{\prime}\left(\theta_{2}\right)+\rho_{\varepsilon}^{\prime}\left(\theta_{1}\right) \rho_{\varepsilon}^{\prime}\left(\theta_{3}\right)\right) \frac{\tau_{\varepsilon}\left(\frac{l_{2}}{2}\right)}{\tau_{\varepsilon}^{\prime}\left(\frac{l_{2}}{2}\right)} \frac{\tau_{\varepsilon}\left(\frac{l_{3}}{2}\right)}{\tau_{\varepsilon}^{\prime}\left(\frac{l_{3}}{2}\right)} \\
& +\left(\rho_{\varepsilon}^{\prime}\left(\theta_{1}\right)+\rho_{\varepsilon}^{\prime}\left(\theta_{2}\right) \rho_{\varepsilon}^{\prime}\left(\theta_{2}\right)\right) \frac{\tau_{\varepsilon}\left(\frac{l_{1}}{2}\right)}{\tau_{\varepsilon}^{\prime}\left(\frac{l_{1}}{2}\right)} \frac{\tau_{\varepsilon}\left(\frac{l_{3}}{2}\right)}{\tau_{\varepsilon}^{\prime}\left(\frac{l_{3}}{2}\right)}
\end{aligned}
$$

(i) If $\varepsilon=-1$, then $\rho_{\varepsilon}^{\prime}\left(\theta_{i}\right)=\cosh \theta_{i}$. Each term in (4-10) and (4-11) is positive. Hence (4-10) and (4-11) are positive.

(ii) If $\varepsilon=1$, then $\rho_{\varepsilon}^{\prime}\left(\theta_{i}\right)=\cos \theta_{i}$. We see that the expression in (4-10) is positive is the same as

$$
\cos \theta_{3} \tanh \frac{l_{2}}{2}+\cos \theta_{2} \tanh \frac{l_{3}}{2}>0 .
$$

By the cosine law, (4-12) is equivalent to

(4-13) $\frac{-\cosh l_{3}+\cosh l_{1} \cosh l_{2}}{\sinh l_{1} \sinh l_{2}} \frac{\sinh l_{2}}{1+\cosh l_{2}}$

$$
+\frac{-\cosh l_{2}+\cosh l_{1} \cosh l_{3}}{\sinh l_{1} \sinh l_{3}} \frac{\sinh l_{3}}{1+\cosh l_{3}}>0 .
$$

By calculation, (4-13) is equivalent to

$$
\cosh l_{1}>\frac{\cosh ^{2} l_{2}+\cosh ^{2} l_{3}+\cosh l_{2}+\cosh l_{3}}{2 \cosh l_{2} \cosh l_{3}+\cosh l_{2}+\cosh l_{3}} .
$$

Since $l_{1}>\left|l_{2}-l_{3}\right|$, therefore $\cosh l_{1}>\cosh \left(l_{2}-l_{3}\right)$. To show (4-14) holds, it is enough to check

(4-15) $\quad \cosh l_{2} \cosh l_{3}-\sinh l_{2} \sinh l_{3} \geq \frac{\cosh ^{2} l_{2}+\cosh ^{2} l_{3}+\cosh l_{2}+\cosh l_{3}}{2 \cosh l_{2} \cosh l_{3}+\cosh l_{2}+\cosh l_{3}}$.

We simplify the notation by introducing $a:=\cosh l_{2}>1, b:=\cosh l_{3}>1$. Then (4-15) is rewritten as

$$
a b-\sqrt{\left(a^{2}-1\right)\left(b^{2}-1\right)} \geq \frac{a^{2}+b^{2}+a+b}{2 a b+a+b} .
$$

(4-16) is equivalent to

$$
a b-\frac{a^{2}+b^{2}+a+b}{2 a b+a+b} \geq \sqrt{\left(a^{2}-1\right)\left(b^{2}-1\right)} .
$$


Since $a>1, b>1$, the left hand side of (4-17) is positive. To show (4-17) holds, we square the two sides and simplify. We have

$$
\begin{aligned}
& \left(a b^{4}+a^{4} b-a^{3} b^{2}-a^{2} b^{3}\right)+\left(a^{4}+b^{4}-2 a^{2} b^{2}\right)+\left(a^{3}+b^{3}-a b^{2}-a^{2} b\right) \geq 0 \\
\Leftrightarrow & (a b+1)\left(a^{3}+b^{3}-a^{2} b-a b^{2}\right)+\left(a^{2}-b^{2}\right)^{2} \geq 0 \\
\Leftrightarrow & (a b+1)(a+b)(a-b)^{2}+\left(a^{2}-b^{2}\right)^{2} \geq 0 .
\end{aligned}
$$

This shows that the expression in (4-10) is positive.

We see that the expression in (4-11) is positive is the same as

$$
\begin{aligned}
-\sin ^{2} \theta_{3} \tanh \frac{l_{1}}{2} \tanh \frac{l_{2}}{2} & +\left(\cos \theta_{2}+\cos \theta_{1} \cos \theta_{3}\right) \tanh \frac{l_{2}}{2} \tanh \frac{l_{3}}{2} \\
& +\left(\cos \theta_{1}+\cos \theta_{2} \cos \theta_{3}\right) \tanh \frac{l_{1}}{2} \tanh \frac{l_{3}}{2}>0 .
\end{aligned}
$$

By the cosine law, (4-18) is equivalent to

$$
\begin{aligned}
-\sin ^{2} \theta_{3} \tanh \frac{l_{1}}{2} \tanh \frac{l_{2}}{2}+\cosh l_{2} & \sin \theta_{1} \sin \theta_{3} \tanh \frac{l_{2}}{2} \tanh \frac{l_{3}}{2} \\
& +\cosh l_{1} \sin \theta_{2} \sin \theta_{3} \tanh \frac{l_{1}}{2} \tanh \frac{l_{3}}{2}>0 .
\end{aligned}
$$

By the sine law, (4-19) is equivalent to

$$
\begin{aligned}
& \left(\cosh l_{1} \sinh l_{2} \tanh \frac{l_{1}}{2}+\cosh l_{2} \sinh l_{1} \tanh \frac{l_{2}}{2}\right) \tanh \frac{l_{3}}{2}>\sinh l_{3} \tanh \frac{l_{1}}{2} \tanh \frac{l_{2}}{2} \\
\Leftrightarrow & \frac{\cosh l_{1} \sinh l_{2}}{\tanh \frac{l_{2}}{2}}+\frac{\cosh l_{2} \sinh l_{1}}{\tanh \frac{l_{1}}{2}}>\frac{\sinh l_{3}}{\tanh \frac{l_{3}}{2}} \\
\Leftrightarrow & \cosh l_{1}\left(1+\cosh l_{2}\right)+\cosh l_{2}\left(1+\cosh l_{1}\right)>1+\cosh l_{3} \\
\Leftrightarrow & \left(\cosh l_{1}+\cosh l_{2}-1\right)+\left(2 \cosh l_{1} \cosh l_{2}-\cosh l_{3}\right)>0 .
\end{aligned}
$$

This is true since $\cosh l_{1}+\cosh l_{2}>1$ and $2 \cosh l_{1} \cosh l_{2}>\cosh l_{1} \cosh l_{2}+$ $\sinh l_{1} \sinh l_{2}=\cosh \left(l_{1}+l_{2}\right)>\cosh l_{3}$. This shows that the expression in (4-11) is positive.

As a corollary of Lemma 4.3 and Lemma 4.4, we obtain the following result.

Corollary 4.5 Given $\left(\phi_{1}, \phi_{2}, \phi_{3}\right) \in I_{\delta}^{3}$, the differential $1-$ form $\sum_{i=1}^{3} \theta_{i} d u_{i}$ is closed in $u\left(\mathcal{M}_{\varepsilon, \delta}\left(\phi_{1}, \phi_{2}, \phi_{3}\right)\right)$. Furthermore, its integration $w(u)=\int^{u} \sum_{i=1}^{3} \theta_{i} d u_{i}$ is a strictly concave function defined in $u\left(\mathcal{M}_{\varepsilon, \delta}\left(\phi_{1}, \phi_{2}, \phi_{3}\right)\right)$ if either

(i) $\varepsilon=-1$ or

(ii) $(\varepsilon, \delta)=(1,1)$ and $\phi_{i} \in\left[\frac{\pi}{2}, \pi\right], i=1,2,3$. 


\section{Furthermore}

$$
\frac{\partial w}{\partial u_{i}}=\theta_{i}
$$

Let's prove the rigidity of circle packing for $\varepsilon=-1$, or $(\varepsilon, \delta)=(1,1)$ and $\Phi: E \rightarrow$ $\left[\frac{\pi}{2}, \pi\right]$. Fix $\Phi: E \rightarrow I_{\delta}$. For each $r \in \mathcal{N}_{\varepsilon, \delta}(\Phi)$, define the function $u$ in terms of $r$ as in (4-5) by

$$
u(v)=-\int_{r(v)}^{\infty} \frac{1}{\tau_{\varepsilon \delta}(t)} d t .
$$

The set of all values of $u$ is $u\left(\mathcal{N}_{\varepsilon, \delta}(\Phi)\right)$ which is an open convex set in $\mathbb{R}^{V}$ due to Lemma 4.2. To be more precise, let us label vertices $V=\left\{v_{1}, \ldots, v_{n}\right\}$ and denote by $\{i, j, k\} \in F$ a generalized triangle in $F$ with vertices $v_{i}, v_{j}, v_{k}$. Let $u_{i}=u\left(v_{i}\right)$. Then $u\left(\mathcal{N}_{\varepsilon, \delta}(\Phi)\right)=\left\{u \in \mathbb{R}^{V} \mid\left(u_{i}, u_{j}, u_{k}\right) \in u\left(\mathcal{M}_{\varepsilon, \delta}\left(\phi_{i}, \phi_{j}, \phi_{k}\right)\right)\right.$ if $\left.\{i, j, k\} \in F\right\}$ is convex since it is the intersection of the convex set $\prod_{\{i, j, k\} \in F} u\left(\mathcal{M}_{\varepsilon, \delta}\left(\phi_{i}, \phi_{j}, \phi_{k}\right)\right)$ with affine spaces.

We now use the function in Corollary 4.5 to introduce a function $W: u\left(\mathcal{N}_{\varepsilon, \delta}(\Phi)\right) \rightarrow \mathbb{R}$ by defining

$$
W(u)=\sum_{\{i, j, k\} \in F} w\left(u_{i}, u_{j}, u_{k}\right)
$$

where the sum is over all triangles in $F$ with vertices $\left\{v_{i}, v_{j}, v_{k}\right\}$. By Corollary 4.5, $W$ is smooth and strictly concave down in $u\left(\mathcal{N}_{\varepsilon, \delta}(\Phi)\right)$ so that

$$
\frac{\partial W}{\partial u_{i}}=\tilde{K}\left(v_{i}\right)
$$

by (4-20) and the definition of $\widetilde{K}$.

By Lemma 2.1, the map $\nabla W: u\left(\mathcal{N}_{\varepsilon, \delta}(\Phi)\right) \rightarrow \mathbb{R}^{V}$ is a smooth embedding. Thus the map from $\left\{r \in \mathcal{N}_{\varepsilon, \delta}(\Phi)\right\}$ to $\left\{\tilde{K} \in \mathbb{R}_{>0}^{V}\right\}$ is a smooth injective map.

\subsection{The image of $\tilde{K}$ for $\varepsilon=-1$}

To prove that the image $X=\{\tilde{K}(v) \mid v \in V\}=\mathbb{R}_{>0}^{V}$ for $\varepsilon=-1$, we will show that $X$ is both open and closed in $\mathbb{R}_{>0}^{V}$. By definition, $X \subset \mathbb{R}_{>0}^{V}$ and $X$ is open due to the injectivity of the map from $\left\{r \in \mathcal{N}_{-1, \delta}(\Phi)\right\}$ to $\mathbb{R}_{>0}^{V}$. It remains to prove that $X$ is closed in $\mathbb{R}_{>0}^{V}$. To this end, let us first establish the following lemma.

Lemma 4.6 Let $\varepsilon=-1$ and $\left(\phi_{i}, \phi_{j}, \phi_{k}\right)$ be given so that $\left(\theta_{i}, \theta_{j}, \theta_{k}\right)=\left(\theta_{i}\left(r_{i}, r_{j}, r_{k}\right)\right.$, $\left.\theta_{j}\left(r_{i}, r_{j}, r_{k}\right), \theta_{k}\left(r_{i}, r_{j}, r_{k}\right)\right)$ are considered as functions of $r_{i}, r_{j}, r_{k} \in J_{-\delta}$. Then

$$
\lim _{r_{k} \rightarrow \infty} \theta_{k}\left(r_{i}, r_{j}, r_{k}\right)=0
$$

and the convergence is uniform. 
Proof We only need to check that for constants $a, b, c \in J_{-\delta}$ the following holds.

(1) If $\lim r_{i}=a, \lim r_{j}=b, \lim r_{k}=\infty$, then $\lim \theta_{k}=0$.

(2) If $\lim r_{i}=c, \lim r_{j}=\infty, \lim r_{k}=\infty$, then $\lim \theta_{k}=0$.

(3) If $\lim r_{i}=\infty, \lim r_{j}=\infty, \lim r_{k}=\infty$, then $\lim \theta_{k}=0$.

The strategy of the proof is the same for all three cases. First, in $\triangle P_{j} P_{k} Q_{i}, \triangle P_{k} P_{i} Q_{j}$, $\triangle P_{i} P_{j} Q_{k}$ of type $(-1,-1, \delta)$, by the cosine law (3-4), the lengths $l_{i}, l_{j}, l_{k}$ can be written as functions of $r_{i}, r_{j}, r_{k}$ :

$$
\begin{aligned}
\cosh l_{i} & =2 \rho_{\delta}^{2}\left(\frac{\phi_{i}}{2}\right) \tau_{-\delta}\left(r_{j}\right) \tau_{-\delta}\left(r_{k}\right)-\frac{1}{2}\left(e^{r_{j}-r_{k}}+e^{r_{k}-r_{j}}\right), \\
\cosh l_{j} & =2 \rho_{\delta}^{2}\left(\frac{\phi_{j}}{2}\right) \tau_{-\delta}\left(r_{k}\right) \tau_{-\delta}\left(r_{i}\right)-\frac{1}{2}\left(e^{r_{k}-r_{i}}+e^{r_{i}-r_{k}}\right), \\
\cosh l_{k} & =2 \rho_{\delta}^{2}\left(\frac{\phi_{k}}{2}\right) \tau_{-\delta}\left(r_{i}\right) \tau_{-\delta}\left(r_{j}\right)-\frac{1}{2}\left(e^{r_{i}-r_{j}}+e^{r_{j}-r_{i}}\right) .
\end{aligned}
$$

When the limits of $r_{i}, r_{j}, r_{k}$ are given, we can find the limits of $l_{i}, l_{j}, l_{k}$. Then in $\triangle P_{i} P_{j} P_{k}$ (of type $(-1,-1,-1)$ ), by the cosine law (3-5), write $\theta_{k}$ as a function of $l_{i}, l_{j}, l_{k}$ :

$$
\cosh \theta_{k}=\frac{\cosh l_{k}+\cosh l_{i} \cosh l_{j}}{\sinh l_{i} \sinh l_{j}} .
$$

Then we find the limit of $\theta_{k}$.

(1) If $\lim r_{i}=a, \lim r_{j}=b, \lim r_{k}=\infty$, then

$$
\begin{aligned}
\lim \cosh l_{i} & =\lim \left(2 \rho_{\delta}^{2}\left(\frac{\phi_{i}}{2}\right) \tau_{-\delta}(b) \frac{1}{2} e^{r_{k}}-\frac{1}{2} e^{r_{k}-b}\right) \\
& =\lim \frac{1}{2} e^{r_{k}}\left(2 \rho_{\delta}^{2}\left(\frac{\phi_{i}}{2}\right) \tau_{-\delta}(b)-1\right) .
\end{aligned}
$$

Since $\cosh l_{i}>0$, we have $2 \rho_{\delta}^{2}\left(\frac{\phi_{i}}{2}\right) \tau_{-\delta}(b)-1>0$. When $\lim r_{k}=\infty$, we have $\lim \cosh l_{i}=\infty$.

By symmetry $\lim \cosh l_{j}=\infty$. Furthermore $\lim \cosh l_{k}$ is finite. Therefore $\lim l_{i}=$ $\lim l_{j}=\infty, \lim l_{k}$ is finite. Hence

$$
\lim \cosh \theta_{k}=\lim \frac{\cosh l_{k}}{\sinh l_{i} \sinh l_{j}}+1=1 .
$$

Therefore $\lim \theta_{k}=0$. 
(2) If $\lim r_{i}=c, \lim r_{j}=\infty, \lim r_{k}=\infty$, then

$$
\begin{aligned}
\lim \cosh l_{i} & =\lim \left(2 \rho_{\delta}^{2}\left(\frac{\phi_{i}}{2}\right) \frac{1}{4} e^{r_{j}+r_{k}}-\frac{1}{2}\left(e^{r_{j}-r_{k}}+e^{r_{k}-r_{j}}\right)\right) \\
& =\lim \frac{1}{2} e^{r_{j}+r_{k}}\left(\rho_{\delta}^{2}\left(\frac{\phi_{i}}{2}\right)-e^{-2 r_{k}}-e^{-2 r_{j}}\right) \\
& =\lim \frac{1}{2} e^{r_{j}+r_{k}} \rho_{\delta}^{2}\left(\frac{\phi_{i}}{2}\right) \\
& =\infty \\
\lim \cosh l_{j} & =\lim \left(2 \rho_{\delta}^{2}\left(\frac{\phi_{j}}{2}\right) \tau_{-\delta}(c) \frac{1}{2} e^{r_{k}}-\frac{1}{2} e^{r_{k}-c}\right) \\
& =\lim e^{r_{k}}\left(\rho_{\delta}^{2}\left(\frac{\phi_{j}}{2}\right) \tau_{-\delta}(c)-\frac{1}{2} e^{-c}\right) \\
& =\infty .
\end{aligned}
$$

Here we use the same argument as in (1).

By the same calculation of $\lim \cosh l_{j}$, we see that $\lim \cosh l_{k}=\lim e^{r_{j}} c_{k}=\infty$ for some constant $c_{k}$. Hence

$$
\begin{aligned}
\lim \cosh \theta_{k} & =\lim \frac{\cosh l_{k}}{\sinh l_{i} \sinh l_{j}}+1 \\
& =\lim \frac{\cosh l_{k}}{\cosh l_{i} \cosh l_{j}}+1 \\
& =\lim \frac{e^{r_{j}} c_{k}}{e^{r_{k}} c_{j} e^{r_{j}+r_{k}} c_{i}}+1 \\
& =1 .
\end{aligned}
$$

Therefore $\lim \theta_{k}=0$.

(3) If $\lim r_{i}=\infty, \lim r_{j}=\infty, \lim r_{k}=\infty$, by the same calculation of $\lim \cosh l_{i}$ in (2), we see that

$\lim \cosh l_{k}=\lim e^{r_{i}+r_{j}} a_{k}, \quad \lim \cosh l_{j}=\lim e^{r_{k}+r_{i}} a_{j}, \quad \lim \cosh l_{i}=\lim e^{r_{j}+r_{k}} a_{i}$ for some constants

$$
\begin{aligned}
\lim \cosh \theta_{k}=\lim \frac{\cosh l_{k}}{\sinh l_{i} \sinh l_{j}}+1 & =\lim \frac{\cosh l_{k}}{\cosh l_{i} \cosh l_{j}}+1 \\
& =\lim \frac{e^{r_{i}+r_{j}} a_{k}}{e^{r_{k}+r_{i}} a_{j} e^{r_{j}+r_{k}} a_{i}}+1=1 .
\end{aligned}
$$

Therefore $\lim \theta_{k}=0$. 
To show that $X$ is closed in $\mathbb{R}_{>0}^{V}$, take a sequence of radius $r^{(m)}$ in $\mathcal{N}_{\Phi,-1}$ such that $\lim _{m \rightarrow \infty} \widetilde{K}^{(m)} \in \mathbb{R}_{>0}^{V}$. To prove closedness, it is sufficient to show that there is a subsequence, say $r^{(m)}$, so that $\lim _{m \rightarrow \infty} r^{(m)}$ is in $\mathcal{N}_{-1, \delta}(\Phi)$.

Suppose otherwise, there is a subsequence, say $r^{(m)}$ so that $\lim _{m \rightarrow \infty} r^{(m)}$ is in the boundary of $\mathcal{N}_{-1, \delta}(\Phi)$. For $\delta= \pm 1$, there are two possibilities that either for some $v \in V, \lim _{m \rightarrow \infty} r^{(m)}(v)=\infty$ or there is an edge $e$ such that $l_{e}^{(m)}=0$. For $\delta=0$, although $r^{(m)}$ 's are allowed to be negative, the limit of $r^{(m)}$ can not be $-\infty$ since when $\phi_{k}$ is given, the condition in Lemma 3.6 (3)

$$
\phi_{k}>\exp \left(r_{i}^{(m)}\right)+\exp \left(r_{j}^{(m)}\right)
$$

implies that $r_{i}^{(m)}$ is bounded away from $-\infty$. Therefore there are only those two possibilities as in case $\delta= \pm 1$.

In the first possibility, by Lemma 4.6 we see each generalized angle incident to the vertex $v$ converges to 0 . Hence $\lim _{m \rightarrow \infty} \widetilde{K}^{(m)}(v)=0$. This contradicts the assumption that $\lim _{m \rightarrow \infty} \tilde{K}^{(m)} \in \mathbb{R}_{>0}^{V}$.

In the second possibility, in a hyperbolic right-angled hexagon with lengths $l_{i}, l_{j}, l_{k}$ and opposite generalized angle $\theta_{i}, \theta_{j}, \theta_{k}$, by the cosine law we see that

$$
\begin{aligned}
\cosh \theta_{j} & =\frac{\cosh l_{j}+\cosh l_{i} \cosh l_{k}}{\sinh l_{i} \sinh l_{k}} \\
& >\frac{\cosh l_{i} \cosh l_{k}}{\sinh l_{i} \sinh l_{k}} \\
& \geq \frac{\cosh l_{i}}{\sinh l_{i}} .
\end{aligned}
$$

Hence we have $\lim _{l_{i} \rightarrow 0} \theta_{j}=\infty$. Hence the generalized discrete curvature containing $\theta_{j}$ converges to $\infty$. This contradicts the assumption that $\lim _{m \rightarrow \infty} \widetilde{K}^{(m)} \in \mathbb{R}_{>0}^{V}$.

Now we have finished the proof of Theorem 1.4.

\section{A proof of Theorem 1.6}

The proof is based on constructing a strictly concave energy function on the space of all generalized hyperbolic triangles of type $(\varepsilon, \varepsilon, \delta)$ so that its gradient is the generalized angles. Then using Lemma 2.1 on injectivity of gradient, we establish Theorem 1.6. 


\subsection{An energy functional on the space of triangles}

Fix a type $(\varepsilon, \varepsilon, \delta) \in\{-1,0,1\}^{3}$. Consider $(\varepsilon, \varepsilon, \delta)$ type generalized hyperbolic triangles whose edge lengths are $l_{1}, l_{2}, l_{3}$ and opposite angles $\theta_{1}, \theta_{2}, \theta_{3}$ so that the angle $\theta_{i}$ faces $l_{i}$ and the type of $\theta_{3}$ angle is $\delta$. For a fixed angle $\theta_{3}$, all values of the two edge lengths $\left(l_{1}, l_{2}\right)$ form the set $\mathcal{D}_{\varepsilon, \delta}\left(\theta_{3}\right)$. For the definition of $\mathcal{D}_{\varepsilon, \delta}\left(\theta_{3}\right)$, see Section 3.

For $h \in \mathbb{R}$, make a change of variables $\left(l_{1}, l_{2}\right)$ to $\left(w_{1}, w_{2}\right)$ and $\left(\theta_{1}, \theta_{2}\right)$ to $\left(a_{1}, a_{2}\right)$ as follows. Let $i=1,2$,

$$
a_{i}=\int_{1}^{\theta_{i}} \rho_{\varepsilon}^{h}(t) d t
$$

where $\rho_{\varepsilon}(t)=\int_{0}^{t} \cos (\sqrt{\varepsilon} x) d x$ and

$$
w_{i}=\int_{1}^{l_{i}} \tau_{\varepsilon \delta}^{h-1}(t) d t
$$

where $\tau_{\varepsilon \delta}(t)=\frac{1}{2} e^{t}-\frac{1}{2} \varepsilon \delta e^{-t}$, as introduced in Section 3. By the construction, the maps $\left(l_{1}, l_{2}\right)$ to $w=\left(w_{1}, w_{2}\right)$ and $\left(\theta_{1}, \theta_{2}\right)$ to $a=\left(a_{1}, a_{2}\right)$ are diffeomorphisms. Thus the cosine law relating $l$ to $\theta$ can be considered, with $\theta_{3}$ fixed, as a smooth map $a=a(w)$ defined on $w\left(\mathcal{D}_{\varepsilon, \delta}\left(\theta_{3}\right)\right)$.

Lemma 5.1 Under the above assumption, for a fixed angle $\theta_{3}$, and any $h \in \mathbb{R}$, the differential 1-form $a_{1} d w_{2}+a_{2} d w_{1}$ is closed in $w\left(\mathcal{D}_{\varepsilon, \delta}\left(\theta_{3}\right)\right)$. Furthermore, the integration

$$
F_{\theta_{3}, h}\left(w_{1}, w_{2}\right)=\int_{(1,1)}^{\left(w_{1}, w_{2}\right)}\left(a_{1} d w_{2}+a_{2} d w_{1}\right)
$$

is strictly concave down in $w\left(\mathcal{D}_{\varepsilon, \delta}\left(\theta_{3}\right)\right)$. In particular

$$
\frac{\partial F_{\theta_{3}, h}}{\partial w_{i}}=a_{j}=\int_{1}^{\theta_{j}} \rho_{\varepsilon}^{h}(t) d t
$$

for $\{i, j\}=\{1,2\}$.

Proof If $\theta_{3}$ is fixed, then $d \theta_{3}=0$. By the derivative cosine law (3-9) we have

$$
\begin{aligned}
\left(\begin{array}{l}
d l_{1} \\
d l_{2}
\end{array}\right) & =\frac{-1}{\sqrt{-\operatorname{det} G_{l}}}\left(\begin{array}{cc}
\tau_{\varepsilon \delta}\left(l_{1}\right) & 0 \\
0 & \tau_{\varepsilon \delta}\left(l_{2}\right)
\end{array}\right)\left(\begin{array}{cc}
\varepsilon & \tau_{\varepsilon \varepsilon}^{\prime}\left(l_{3}\right) \\
\tau_{\varepsilon \varepsilon}^{\prime}\left(l_{3}\right) & \varepsilon
\end{array}\right)\left(\begin{array}{l}
d \theta_{1} \\
d \theta_{2}
\end{array}\right) \\
& =\frac{-1}{\sqrt{-\operatorname{det} G_{l}}}\left(\begin{array}{cc}
\tau_{\varepsilon \delta}\left(l_{1}\right) & 0 \\
0 & \tau_{\varepsilon \delta}\left(l_{2}\right)
\end{array}\right)\left(\begin{array}{cc}
\tau_{\varepsilon \varepsilon}^{\prime}\left(l_{3}\right) & \varepsilon \\
\varepsilon & \tau_{\varepsilon \varepsilon}^{\prime}\left(l_{3}\right)
\end{array}\right)\left(\begin{array}{l}
d \theta_{2} \\
d \theta_{1}
\end{array}\right) .
\end{aligned}
$$


Since $d w_{i}=\tau_{\varepsilon \delta}^{h-1}\left(l_{i}\right) d l_{i}, d a_{i}=\rho_{\varepsilon}^{h}\left(\theta_{i}\right) d \theta_{i}$, for $i=1,2$, then

$$
\begin{aligned}
\left(\begin{array}{l}
d w_{1} \\
d w_{2}
\end{array}\right) & =\frac{-1}{\sqrt{-\operatorname{det} G_{l}}}\left(\begin{array}{cc}
\tau_{\varepsilon \delta}^{h}\left(l_{1}\right) & 0 \\
0 & \tau_{\varepsilon \delta}^{h}\left(l_{2}\right)
\end{array}\right)\left(\begin{array}{cc}
\tau_{\varepsilon \varepsilon}^{\prime}\left(l_{3}\right) & \varepsilon \\
\varepsilon & \tau_{\varepsilon \varepsilon}^{\prime}\left(l_{3}\right)
\end{array}\right) \\
& \left(\begin{array}{cc}
\rho_{\varepsilon}^{-h}\left(\theta_{2}\right) & 0 \\
0 & \rho_{\varepsilon}^{-h}\left(\theta_{1}\right)
\end{array}\right)\left(\begin{array}{l}
d a_{2} \\
d a_{1}
\end{array}\right) \\
& =\frac{-1}{\sqrt{-\operatorname{det} G_{l}}} A\left(\begin{array}{l}
d a_{2} \\
d a_{1}
\end{array}\right) .
\end{aligned}
$$

Since $A_{12}=\tau_{\varepsilon \delta}^{h}\left(l_{1}\right) \varepsilon \rho_{\varepsilon}^{-h}\left(\theta_{1}\right)=\tau_{\varepsilon \delta}^{h}\left(l_{2}\right) \varepsilon \rho_{\varepsilon}^{-h}\left(\theta_{2}\right)=A_{21}$ by the sine law (3-6), the matrix $A$ is symmetric. Thus the differential 1 -form $a_{1} d w_{2}+a_{2} d w_{1}$ is closed. Therefore the function $F_{\theta_{3}, h}\left(w_{1}, w_{2}\right)$ is well defined.

The above calculation shows that the Hessian of the function $F_{\theta_{3}, h}\left(w_{1}, w_{2}\right)$ is the matrix $-\sqrt{-\operatorname{det} G_{l}} A^{-1}$. To show the function $F_{\theta_{3}, h}\left(w_{1}, w_{2}\right)$ is strictly concave down, we need to check that $A^{-1}$ is positive definite. It is equivalent to show that $A$ is positive definite. By forgetting the two diagonal matrices, it is enough to show

$$
B=\left(\begin{array}{cc}
\tau_{\varepsilon \varepsilon}^{\prime}\left(l_{3}\right) & \varepsilon \\
\varepsilon & \tau_{\varepsilon \varepsilon}^{\prime}\left(l_{3}\right)
\end{array}\right)
$$

is positive definite. Since $\tau_{\varepsilon \varepsilon}^{\prime}\left(l_{3}\right)=1 / 2\left(e^{l_{3}}+\epsilon^{2} e^{-l_{3}}\right)>0$ and

$$
\operatorname{det} B=\tau_{\varepsilon \varepsilon}^{\prime 2}\left(l_{3}\right)-\varepsilon^{2}=\left(\frac{1}{2} e^{l_{3}}+\frac{1}{2} \varepsilon \varepsilon e^{-l_{3}}\right)^{2}-\varepsilon^{2}=\left(\frac{1}{2} e^{l_{3}}-\frac{1}{2} \varepsilon \varepsilon e^{-l_{3}}\right)^{2}>0,
$$

the matrix $B$ is positive definite.

\subsection{A proof of Theorem 1.6}

Now the proof of Theorem 1.6 follows from the routine variational framework. Let us recall the set up in Section 1.5. Suppose $(\Sigma, G)$ is a cell decomposed surface so that the sets of all vertices, edges and 2-cells are $V, E, F$ respectively. The dual decomposition is $G^{*}$ with vertices $V^{*}(\cong F)$. Elements in $V^{*}$ are denoted by $f^{*}$ where $f \in F$. A quadrilateral $\left(v, v^{\prime}, f^{*}, f^{\prime *}\right) \in V \times V \times V^{*} \times V^{*}$ in $\Sigma$ satisfies $v v^{\prime} \in E, f>v v^{\prime}$ and $f^{\prime}>v v^{\prime}$.

Now fix a type $(\varepsilon, \varepsilon, \delta)$ and a function $\theta: E \rightarrow \stackrel{\circ}{I}_{\delta}$. The set of all circle pattern metrics is $\mathcal{E}_{\varepsilon, \delta}(\theta)=\left\{r \in\left(J_{\varepsilon \delta}\right)^{V^{*}} \mid\left(r_{i}, r_{j}\right) \in \mathcal{D}_{\varepsilon, \delta}\left(\theta\left(v_{i} v_{j}\right)\right)\right.$ whenever $f_{i}, f_{j}$ share an edge $\}$. For any circle pattern metric $r \in \mathcal{E}_{\varepsilon, \delta}(\theta)$ and any quadrilateral $\left(v, v^{\prime}, f^{*}, f^{\prime *}\right) \in$ $V \times V \times V^{*} \times V^{*}$ where $v v^{\prime} \in E, f>v v^{\prime}$ and $f^{\prime}>v v^{\prime}$, construct a type $(\varepsilon, \varepsilon, \delta)$ generalized hyperbolic triangle $\triangle f^{*} f^{*} v$ so that the lengths of $f^{*} v$ and $f^{*} v$ are 
$r\left(f^{*}\right), r\left(f^{*}\right)$, and the generalized angle at $v$ is $\theta\left(v v^{\prime}\right)$ of type $\delta$. Realize the quadrilateral $\left(v, v^{\prime}, f^{*}, f^{\prime *}\right)$ geometrically as the metric double of $\triangle f^{*} v f^{\prime *}$ along the edge $f^{*} f^{\prime *}$. Now isometrically glue all these geometric quadrilaterals along edges. The result is a polyhedral surface. Recall that for $h \in \mathbb{R}$, the $K_{h}$-curvature of $r$

$$
K_{h}: V^{*} \rightarrow \mathbb{R}
$$

is defined by (1-3).

For $h \in \mathbb{R}$, make a change of parameter from $r \in \mathcal{E}_{\varepsilon, \delta}(\theta)$ to $w=w(r) \in w\left(\mathcal{E}_{\varepsilon, \delta}(\theta)\right)$ so that $w(r)(x)=\int_{1}^{r(x)} \tau_{\varepsilon \delta}^{h-1}(s) d s$ is given by (5-2). We now use Lemma 5.1 to construct a smooth strictly concave function $W: w\left(\mathcal{E}_{\varepsilon, \delta}(\theta)\right) \rightarrow \mathbb{R}$ so that $\left.\nabla W\right|_{w}$ is the generalized curvature $K_{h}$ of $r$ where $w=w(r)$. Then using Lemma 2.1, we see that Theorem 1.6 follows.

Here is the construction. For $w=w(r) \in w\left(\mathcal{E}_{\varepsilon, \delta}(\theta)\right)$ and for each quadrilateral $\left(v, v^{\prime}, f^{*}, f^{\prime *}\right)$ in $\Sigma$, we define the F-energy of it in $r$ metric to be

$$
F_{\theta\left(v v^{\prime}\right), h}\left(w\left(r\left(f^{*}\right)\right), w\left(r\left(f^{\prime *}\right)\right)\right)
$$

where $F_{\theta, h}$ is given by Lemma 5.1. The function $W: w=w(r) \in w\left(\mathcal{E}_{\varepsilon, \delta}(\theta)\right) \rightarrow \mathbb{R}$ is the sum of F-energies of all quadrilateral $\left(v, v^{\prime}, f^{*}, f^{\prime *}\right)$ in $r$ metric. By the construction, $W$ is smooth and strictly concave. By (5-3) we have

$$
\left.\nabla W\right|_{w}=\left.K_{h}\right|_{r}
$$

where $w=w(r)$. This ends the proof of Theorem 1.6.

\section{Appendix A Formulas of cosine and sine laws}

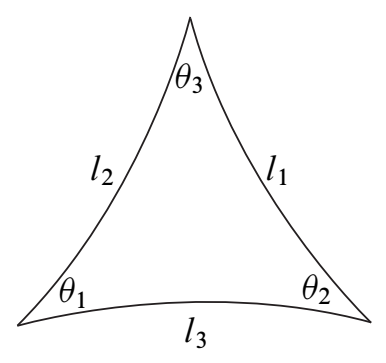

$$
\begin{aligned}
& \text { For }\{i, j, k\}=\{1,2,3\}, \\
& \begin{aligned}
\cosh l_{i} & =\frac{\cos \theta_{i}+\cos \theta_{j} \cos \theta_{k}}{\sin \theta_{j} \sin \theta_{k}}, \\
\cos \theta_{i} & =\frac{-\cosh l_{i}+\cosh l_{j} \cosh l_{k}}{\sinh l_{j} \sinh l_{k}}, \\
\frac{\sin \theta_{1}}{\sinh l_{1}} & =\frac{\sin \theta_{2}}{\sinh l_{2}}=\frac{\sin \theta_{3}}{\sinh l_{3}} .
\end{aligned}
\end{aligned}
$$




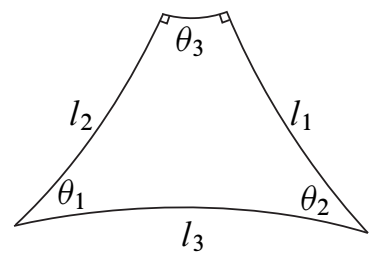

$$
\text { For } \begin{aligned}
\{i, j\} & =\{1,2\}, \\
\sinh l_{i} & =\frac{\cos \theta_{i}+\cos \theta_{j} \cosh \theta_{3}}{\sin \theta_{j} \sinh \theta_{3}}, \\
\cosh l_{3} & =\frac{\cosh \theta_{3}+\cos \theta_{1} \cos \theta_{2}}{\sin \theta_{1} \sin \theta_{2}}, \\
\cos \theta_{i} & =\frac{-\sinh l_{i}+\sinh l_{j} \cosh l_{3}}{\cosh l_{j} \sinh l_{3}}, \\
\cosh \theta_{3} & =\frac{\cosh l_{3}+\sinh l_{1} \sinh l_{2}}{\cosh l_{1} \cosh l_{2}}, \\
\frac{\sin \theta_{1}}{\cosh l_{1}} & =\frac{\sin \theta_{2}}{\cosh l_{2}}=\frac{\sinh \theta_{3}}{\sinh l_{3}}
\end{aligned}
$$

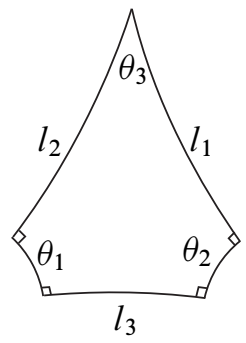

$$
\text { For } \begin{aligned}
\{i, j\} & =\{1,2\}, \\
\sinh l_{i} & =\frac{\cosh \theta_{i}+\cosh \theta_{j} \cos \theta_{3}}{\sinh \theta_{j} \sin \theta_{3}}, \\
\cosh l_{3} & =\frac{\cos \theta_{3}+\cosh \theta_{1} \cosh \theta_{2}}{\sinh \theta_{1} \sinh \theta_{2}}, \\
\cos \theta_{i} & =\frac{\sinh l_{i}+\sinh l_{j} \cosh l_{3}}{\cosh l_{j} \sinh l_{3}}, \\
\cos \theta_{3} & =\frac{-\cosh l_{3}+\sinh l_{1} \sinh l_{2}}{\cosh l_{1} \cosh l_{2}}, \\
\frac{\sinh \theta_{1}}{\cosh l_{1}} & =\frac{\sinh \theta_{2}}{\cosh l_{2}}=\frac{\sin \theta_{3}}{\sinh l_{3}} .
\end{aligned}
$$

For $\{i, j, k\}=\{1,2,3\}$,

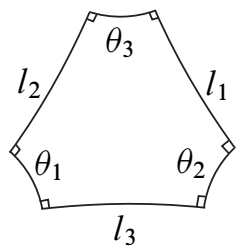

$$
\begin{aligned}
\cosh l_{i} & =\frac{\cosh \theta_{i}+\cosh \theta_{j} \cosh \theta_{k}}{\sinh \theta_{j} \sinh \theta_{k}}, \\
\cosh \theta_{i} & =\frac{\cosh l_{i}+\cosh l_{j} \cosh l_{k}}{\sinh l_{j} \sinh l_{k}}, \\
\frac{\sinh \theta_{1}}{\sinh l_{1}} & =\frac{\sinh \theta_{2}}{\sinh l_{2}}=\frac{\sinh \theta_{3}}{\sinh l_{3}} .
\end{aligned}
$$




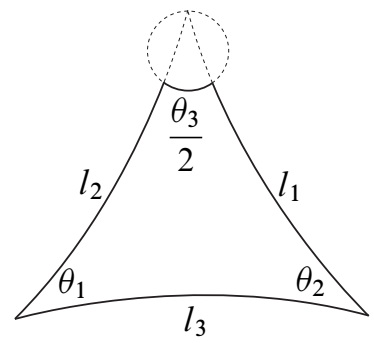

For $\{i, j\}=\{1,2\}$,

$$
\begin{array}{rlrl}
\frac{e^{l_{i}}}{2} & =\frac{\cos \theta_{i}+\cos \theta_{j}}{\theta_{3} \sin \theta_{j}}, & \cos \theta_{i} & =\frac{-e^{l_{i}}+e^{l_{j}} \cosh l_{3}}{e^{l_{j}} \sinh l_{3}}, \\
\cosh l_{3} & =\frac{1+\cos \theta_{1} \cos \theta_{2}}{\sin \theta_{1} \sin \theta_{2}}, & \frac{\theta_{3}^{2}}{2}=\frac{\cosh l_{3}-\cosh \left(l_{1}-l_{2}\right)}{\frac{e^{l_{1}+l_{2}}}{4}}, \\
\frac{\sin \theta_{1}}{\frac{e^{l_{1}}}{2}}=\frac{\sin \theta_{2}}{\frac{e^{l_{2}}}{2}}=\frac{\theta_{3}}{\sinh l_{3}} & & &
\end{array}
$$

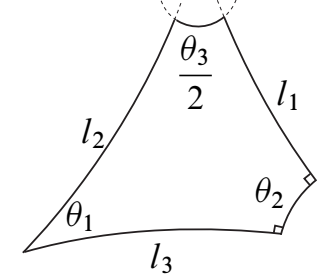

$$
\begin{aligned}
\frac{e^{l_{1}}}{2} & =\frac{\cos \theta_{1}+\cosh \theta_{2}}{\theta_{3} \sinh \theta_{2}} & \cos \theta_{1} & =\frac{-e^{l_{1}}+e^{l_{2}} \sinh l_{3}}{e^{l_{2}} \cosh l_{3}} \\
\frac{e^{l_{2}}}{2} & =\frac{\cosh \theta_{2}+\cos \theta_{1}}{\theta_{3} \sin \theta_{1}} & \cosh \theta_{2} & =\frac{e^{l_{2}}+e^{l_{1}} \sinh l_{3}}{e^{l_{1}} \cosh l_{3}} \\
\sinh l_{3} & =\frac{1+\cos \theta_{1} \cosh \theta_{2}}{\sin \theta_{1} \sinh \theta_{2}} & \frac{\theta_{3}^{2}}{2} & =\frac{\sinh l_{3}+\sinh \left(l_{2}-l_{1}\right)}{\frac{e^{l_{1}+l_{2}}}{4}}
\end{aligned}
$$$$
\frac{\theta_{3}^{2}}{2}=\frac{\sinh l_{3}+\sinh \left(l_{2}-l_{1}\right)}{\frac{e^{l_{1}+l_{2}}}{4}}
$$$$
\frac{\sin \theta_{1}}{\frac{e^{l_{1}}}{2}}=\frac{\sinh \theta_{2}}{\frac{e^{l_{2}}}{2}}=\frac{\theta_{3}}{\cosh l_{3}}
$$ 


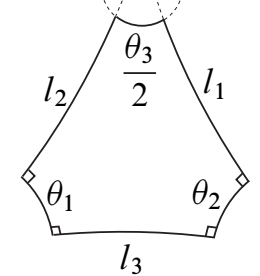

For $\{i, j\}=\{1,2\}$,

$$
\begin{array}{rlrl}
\frac{e^{l_{i}}}{2} & =\frac{\cosh \theta_{i}+\cosh \theta_{j}}{\theta_{3} \sinh \theta_{j}}, & \cosh \theta_{i} & =\frac{e^{l_{i}}+e^{l_{j}} \cosh l_{3}}{e^{l_{j}} \sinh l_{3}}, \\
\cosh l_{3} & =\frac{1+\cosh \theta_{1} \cosh \theta_{2}}{\sinh \theta_{1} \sinh \theta_{2}}, & \frac{\theta_{3}^{2}}{2}=\frac{\cosh l_{3}+\cosh \left(l_{1}-l_{2}\right)}{\frac{e^{l_{1}+l_{2}}}{4}}, \\
\frac{\sinh \theta_{1}}{\frac{e^{l_{1}}}{2}} & =\frac{\sinh \theta_{2}}{\frac{e^{l_{2}}}{2}}=\frac{\theta_{3}}{\sinh l_{3}} . &
\end{array}
$$

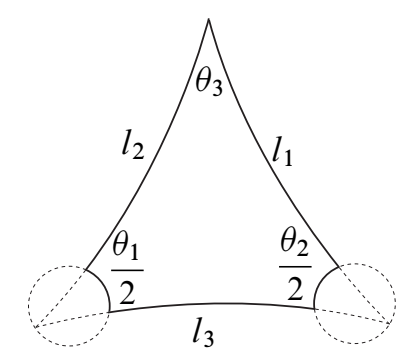

For $\{i, j\}=\{1,2\}$,

$$
\begin{array}{rlrl}
\frac{e^{l_{i}}}{2} & =\frac{1+\cos \theta_{3}}{\theta_{j} \sin \theta_{3}}, & \frac{\theta_{i}^{2}}{4} & =\frac{e^{l_{i}}-e^{l_{3}-l_{j}}}{e^{l_{j}+l_{3}}}, \\
\frac{e^{l_{3}}}{2} & =\frac{1+\cos \theta_{3}}{\theta_{1} \theta_{2}}, & \sin ^{2} \frac{\theta_{3}}{2} & =e^{l_{3}-l_{1}-l_{2}}, \\
\frac{\theta_{1}}{e^{l_{1}}} & =\frac{\theta_{2}}{e^{l_{2}}}=\frac{\sin \theta_{3}}{e^{l_{3}}} . &
\end{array}
$$




$$
\begin{aligned}
\text { For }\{i, j\}=\{1,2\} & , \\
\frac{e^{l_{i}}}{2} & =\frac{1+\cosh \theta_{3}}{\theta_{j} \sinh \theta_{3}}, \\
\frac{e^{l_{3}}}{2} & =\frac{1+\cosh \theta_{3}}{\theta_{1} \theta_{2}}, \\
\frac{\theta_{i}^{2}}{4} & =\frac{e^{l_{i}}+e^{l_{3}-l_{j}}}{e^{l_{j}+l_{3}}}, \\
\sinh ^{2} \frac{\theta_{3}}{2} & =e^{l_{3}-l_{1}-l_{2}}, \\
\frac{\theta_{1}}{e^{l_{1}}} & =\frac{\theta_{2}}{e^{l_{2}}}=\frac{\sinh \theta_{3}}{e^{l_{3}}} .
\end{aligned}
$$

For $\{i, j, k\}=\{1,2,3\}$,

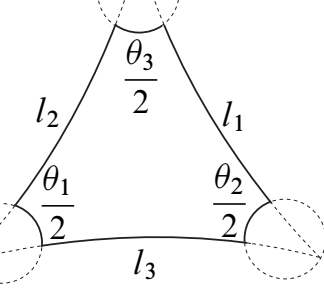

$$
\begin{aligned}
\frac{e^{l_{i}}}{2} & =\frac{2}{\theta_{j} \theta_{k}}, \\
\frac{\theta_{i}^{2}}{4} & =e^{l_{i}-l_{j}-l_{k}}, \\
\frac{\theta_{1}}{e^{l_{1}}} & =\frac{\theta_{2}}{e^{l_{2}}}=\frac{\theta_{3}}{e^{l_{3}}} .
\end{aligned}
$$

\section{Appendix B Proofs of Lemma 3.3 and Lemma 3.5}

For simplicity, we abuse the notation. Let

$$
\begin{array}{ll}
g\left(\theta_{i}\right):=\rho_{\varepsilon_{i}}\left(\theta_{i}\right), & g^{\prime}\left(\theta_{i}\right):=\rho_{\varepsilon_{i}}^{\prime}\left(\theta_{i}\right), \\
f\left(l_{k}\right):=\tau_{\varepsilon_{i} \varepsilon_{j}}\left(l_{k}\right), & f^{\prime}\left(l_{k}\right):=\tau_{\varepsilon_{i} \varepsilon_{j}}^{\prime}\left(l_{k}\right),
\end{array}
$$

for $\{i, j, k\}=\{1,2,3\}$, where $g, f$ represent functions which depend on the type of generalized vertices.

In this simplified notation we have

$$
\begin{aligned}
-\operatorname{det} G_{l} & =\operatorname{det}\left(\begin{array}{ccc}
\varepsilon_{1} & f^{\prime}\left(l_{3}\right) & f^{\prime}\left(l_{2}\right) \\
f^{\prime}\left(l_{3}\right) & \varepsilon_{2} & f^{\prime}\left(l_{1}\right) \\
f^{\prime}\left(l_{2}\right) & f^{\prime}\left(l_{1}\right) & \varepsilon_{3}
\end{array}\right) \\
& =\varepsilon_{1} \varepsilon_{2} \varepsilon_{3}+2 f^{\prime}\left(l_{1}\right) f^{\prime}\left(l_{2}\right) f^{\prime}\left(l_{3}\right)-\varepsilon_{1} f^{\prime 2}\left(l_{1}\right)-\varepsilon_{2} f^{\prime 2}\left(l_{2}\right)-\varepsilon_{3} f^{\prime 2}\left(l_{3}\right) .
\end{aligned}
$$


If $\varepsilon_{3}=0$, then $g\left(\theta_{3}\right)=\theta_{3}, f\left(l_{1}\right)=\frac{e^{l_{1}}}{2}, f\left(l_{2}\right)=\frac{e^{l_{2}}}{2}$. By the cosine law (3-4), we have

$$
\frac{\theta_{3}^{2}}{2}=\frac{f^{\prime}\left(l_{3}\right)-\frac{1}{2} \varepsilon_{1} e^{l_{1}-l_{2}}-\frac{1}{2} \varepsilon_{2} e^{l_{2}-l_{1}}}{f\left(l_{1}\right) f\left(l_{2}\right)} .
$$

Thus the negative of the right hand side of (3-7) is

$$
\begin{aligned}
f^{2}\left(l_{1}\right) f^{2}\left(l_{2}\right) g^{2}\left(\theta_{3}\right) & =f^{2}\left(l_{1}\right) f^{2}\left(l_{2}\right) \theta_{3}^{2} \\
& =f^{2}\left(l_{1}\right) f^{2}\left(l_{2}\right) \frac{2 f^{\prime}\left(l_{3}\right)-\varepsilon_{1} e^{l_{1}-l_{2}}-\varepsilon_{2} e^{l_{2}-l_{1}}}{f\left(l_{1}\right) f\left(l_{2}\right)} \\
& =f\left(l_{1}\right) f\left(l_{2}\right)\left(2 f^{\prime}\left(l_{3}\right)-\varepsilon_{1} e^{l_{1}-l_{2}}-\varepsilon_{2} e^{l_{2}-l_{1}}\right) \\
& =\frac{e^{l_{1}}}{2} \frac{e^{l_{2}}}{2}\left(2 f^{\prime}\left(l_{3}\right)-\varepsilon_{1} e^{l_{1}-l_{2}}-\varepsilon_{2} e^{l_{2}-l_{1}}\right) \\
& =2 \frac{e^{l_{1}}}{2} \frac{e^{l_{2}}}{2} f^{\prime}\left(l_{3}\right)-\varepsilon_{1} \frac{e^{2 l_{1}}}{4}-\varepsilon_{2} \frac{e^{2 l_{2}}}{4} \\
& =-\operatorname{det} G_{l} .
\end{aligned}
$$

If $\varepsilon_{3}= \pm 1$, then $g^{2}\left(\theta_{3}\right)=\varepsilon_{3}\left(1-g^{\prime 2}\left(\theta_{3}\right)\right)$. And we have

(B-1) $f^{2}\left(l_{i}\right)-f^{\prime 2}\left(l_{i}\right)=\left(\frac{1}{2} e^{l}-\frac{1}{2} \varepsilon_{j} \varepsilon_{k} e^{-l}\right)^{2}-\left(\frac{1}{2} e^{l}+\frac{1}{2} \varepsilon_{j} \varepsilon_{k} e^{-l}\right)^{2}=-\varepsilon_{j} \varepsilon_{k}$.

Thus the negative of the right hand side of (3-7) is

$$
\begin{aligned}
f^{2}\left(l_{1}\right) f^{2}\left(l_{2}\right) g^{2}\left(\theta_{3}\right) & =f^{2}\left(l_{1}\right) f^{2}\left(l_{2}\right)\left(1-g^{\prime 2}\left(\theta_{3}\right)\right) \\
& =\varepsilon_{3}\left(f^{2}\left(l_{1}\right) f^{2}\left(l_{2}\right)-\left(-\varepsilon_{3} f^{\prime}\left(l_{3}\right)+f^{\prime}\left(l_{1}\right) f^{\prime}\left(l_{2}\right)\right)^{2}\right) \\
& =\varepsilon_{3}\left(\left(f^{\prime 2}\left(l_{1}\right)-\varepsilon_{2} \varepsilon_{3}\right)\left(f^{\prime 2}\left(l_{2}\right)-\varepsilon_{3} \varepsilon_{1}\right)\right. \\
& \left.-\left(-\varepsilon_{3} f^{\prime}\left(l_{3}\right)+f^{\prime}\left(l_{1}\right) f^{\prime}\left(l_{2}\right)\right)^{2}\right) \\
& =\varepsilon_{3}\left(\varepsilon_{1} \varepsilon_{2} \varepsilon_{3}^{2}+2 \varepsilon_{3} f^{\prime}\left(l_{1}\right) f^{\prime}\left(l_{2}\right) f^{\prime}\left(l_{3}\right)\right. \\
& \left.\quad-\varepsilon_{1} \varepsilon_{3} f^{\prime 2}\left(l_{1}\right)-\varepsilon_{2} \varepsilon_{3} f^{\prime 2}\left(l_{2}\right)-\varepsilon_{3}^{2} f^{\prime 2}\left(l_{3}\right)\right) \\
& =-\operatorname{det} G_{l} .
\end{aligned}
$$

The second equality is due to the cosine law, the third equality is due to (B-1) and the last equality is due to $\varepsilon_{3}= \pm 1$. 
And we have

$$
\begin{aligned}
-\operatorname{det} G_{\theta} & =\operatorname{det}\left(\begin{array}{ccc}
-1 & g^{\prime}\left(\theta_{3}\right) & g^{\prime}\left(\theta_{2}\right) \\
g^{\prime}\left(\theta_{3}\right) & -1 & g^{\prime}\left(\theta_{1}\right) \\
g^{\prime}\left(\theta_{2}\right) & g^{\prime}\left(\theta_{1}\right) & -1
\end{array}\right) \\
& =-1+2 g^{\prime}\left(\theta_{1}\right) g^{\prime}\left(\theta_{2}\right) g^{\prime}\left(\theta_{3}\right)+g^{\prime 2}\left(\theta_{1}\right)+g^{\prime 2}\left(\theta_{2}\right)+g^{\prime 2}\left(\theta_{3}\right) .
\end{aligned}
$$

Notice that we have

$$
g^{\prime 2}\left(\theta_{i}\right)+\varepsilon_{i} g^{2}\left(\theta_{i}\right)=1
$$

Thus negative of the right hand side of (3-8) is

$$
\begin{aligned}
g^{2}\left(\theta_{1}\right) g^{2}\left(\theta_{2}\right) f^{2}\left(l_{3}\right) & =g^{2}\left(\theta_{1}\right) g^{2}\left(\theta_{2}\right)\left(f^{\prime 2}\left(l_{3}\right)-\varepsilon_{1} \varepsilon_{2}\right) \\
& =\left(g^{\prime}\left(\theta_{3}\right)+g^{\prime}\left(\theta_{1}\right) g^{\prime}\left(\theta_{2}\right)\right)^{2}-g^{2}\left(\theta_{1}\right) g^{2}\left(\theta_{2}\right) \varepsilon_{1} \varepsilon_{2} \\
& =\left(g^{\prime}\left(\theta_{3}\right)+g^{\prime}\left(\theta_{1}\right) g^{\prime}\left(\theta_{2}\right)\right)^{2}-\left(1-g^{\prime 2}\left(\theta_{1}\right)\right)\left(1-g^{\prime 2}\left(\theta_{2}\right)\right) \\
& =-\operatorname{det} G_{\theta} .
\end{aligned}
$$

The second equality is due to the cosine law and the third equality is due to (B-2).

We can prove either one of the derivative cosine law (3-9) and (3-10). The other one will be a corollary due to Lemma 3.4. For example, if (3-9) is true, we have

$$
\left(\begin{array}{l}
d l_{1} \\
d l_{2} \\
d l_{3}
\end{array}\right)=M G_{l}\left(\begin{array}{l}
d \theta_{1} \\
d \theta_{2} \\
d \theta_{3}
\end{array}\right) .
$$

It is equivalent to

$$
\left(\begin{array}{l}
d \theta_{1} \\
d \theta_{2} \\
d \theta_{3}
\end{array}\right)=\left(M G_{l}\right)^{-1}\left(\begin{array}{l}
d l_{1} \\
d l_{2} \\
d l_{3}
\end{array}\right) .
$$

By Lemma 3.4, $\left(M G_{l}\right)^{-1}=M G_{\theta}$. Thus we obtained (3-10):

$$
\left(\begin{array}{l}
d \theta_{1} \\
d \theta_{2} \\
d \theta_{3}
\end{array}\right)=M G_{\theta}\left(\begin{array}{l}
d l_{1} \\
d l_{2} \\
d l_{3}
\end{array}\right) .
$$

In the following, we give a proof of (3-9). By the cosine law (3-3) we have

$$
f^{\prime}\left(l_{i}\right) g\left(\theta_{j}\right) g\left(\theta_{k}\right)=g^{\prime}\left(\theta_{i}\right)+g^{\prime}\left(\theta_{j}\right) g^{\prime}\left(\theta_{k}\right) .
$$


After differentiating the two sides we have

$$
\begin{aligned}
& f^{\prime \prime}\left(l_{i}\right) g\left(\theta_{j}\right) g\left(\theta_{k}\right) d l_{i}+f^{\prime}\left(l_{i}\right) g^{\prime}\left(\theta_{j}\right) g\left(\theta_{k}\right) d \theta_{j}+f^{\prime}\left(l_{i}\right) g\left(\theta_{j}\right) g^{\prime}\left(\theta_{k}\right) d \theta_{k} \\
& =g^{\prime \prime}\left(\theta_{i}\right) d \theta_{i}+g^{\prime \prime}\left(\theta_{j}\right) g^{\prime}\left(\theta_{k}\right) d \theta_{j}+g^{\prime}\left(\theta_{j}\right) g^{\prime \prime}\left(\theta_{k}\right) d \theta_{k}
\end{aligned}
$$

which is equivalent to

(B-3) $f^{\prime \prime}\left(l_{i}\right) g\left(\theta_{j}\right) g\left(\theta_{k}\right) d l_{i}$

$$
\begin{aligned}
&=g^{\prime \prime}\left(\theta_{i}\right) d \theta_{i}+\left(g^{\prime \prime}\left(\theta_{j}\right) g^{\prime}\left(\theta_{k}\right)-f^{\prime}\left(l_{i}\right) g^{\prime}\left(\theta_{j}\right) g\left(\theta_{k}\right)\right) d \theta_{j} \\
&+\left(g^{\prime}\left(\theta_{j}\right) g^{\prime \prime}\left(\theta_{k}\right)-f^{\prime}\left(l_{i}\right) g\left(\theta_{j}\right) g^{\prime}\left(\theta_{k}\right)\right) d \theta_{k} .
\end{aligned}
$$

By the cosine law (3-3), the coefficient of $d \theta_{j}$ in (B-3) is

$$
g^{\prime \prime}\left(\theta_{j}\right) g^{\prime}\left(\theta_{k}\right)-f^{\prime}\left(l_{i}\right) g^{\prime}\left(\theta_{j}\right) g\left(\theta_{k}\right)
$$

$$
\begin{aligned}
& =g^{\prime \prime}\left(\theta_{j}\right) g^{\prime}\left(\theta_{k}\right)-\frac{g^{\prime}\left(\theta_{i}\right)+g^{\prime}\left(\theta_{j}\right) g^{\prime}\left(\theta_{k}\right)}{g\left(\theta_{j}\right) g\left(\theta_{k}\right)} g^{\prime}\left(\theta_{j}\right) g\left(\theta_{k}\right) \\
& =\frac{1}{g\left(\theta_{j}\right)}\left(\left(g\left(\theta_{j}\right) g^{\prime \prime}\left(\theta_{j}\right)-g^{\prime 2}\left(\theta_{j}\right)\right) g^{\prime}\left(\theta_{k}\right)-g^{\prime}\left(\theta_{i}\right) g^{\prime}\left(\theta_{j}\right)\right) .
\end{aligned}
$$

For $g(\theta)=\sin \theta$, or $\sinh \theta$, or $\theta$, we always have

$$
g\left(\theta_{j}\right) g^{\prime \prime}\left(\theta_{j}\right)-g^{\prime 2}\left(\theta_{j}\right)=-1 .
$$

Hence (B-4) is

$$
\frac{1}{g\left(\theta_{j}\right)}\left(-g^{\prime}\left(\theta_{k}\right)-g^{\prime}\left(\theta_{i}\right) g^{\prime}\left(\theta_{j}\right)\right)=-g\left(\theta_{i}\right) f\left(l_{k}\right)
$$

due to the cosine law (3-3). By symmetry, the similar formula holds for the coefficient of $d \theta_{k}$. Hence (B-3) is

$$
f^{\prime \prime}\left(l_{i}\right) g\left(\theta_{j}\right) g\left(\theta_{k}\right) d l_{i}=g^{\prime \prime}\left(\theta_{i}\right) d \theta_{i}-g\left(\theta_{i}\right) f\left(l_{k}\right) d \theta_{j}-g\left(\theta_{i}\right) f\left(l_{j}\right) d \theta_{k} .
$$

By the definition of $f$, we have $f^{\prime \prime}=f$. Thus

$$
\begin{aligned}
d l_{i} & =\frac{-g\left(\theta_{i}\right)}{f\left(l_{i}\right) g\left(\theta_{j}\right) g\left(\theta_{k}\right)}\left(-\frac{g^{\prime \prime}\left(\theta_{i}\right)}{g\left(\theta_{i}\right)} d \theta_{i}+f\left(l_{k}\right) d \theta_{j}+f\left(l_{j}\right) d \theta_{k}\right) \\
& =\frac{-g\left(\theta_{i}\right)}{f\left(l_{i}\right) g\left(\theta_{j}\right) g\left(\theta_{k}\right)}\left(\varepsilon_{i} d \theta_{i}+f\left(l_{k}\right) d \theta_{j}+f\left(l_{j}\right) d \theta_{k}\right) .
\end{aligned}
$$

This proves (3-9). 


\section{References}

[1] A I Bobenko, B A Springborn, Variational principles for circle patterns and Koebe's theorem, Trans. Amer. Math. Soc. 356 (2004) 659-689 MR2022715

[2] W Brägger, Kreispackungen und Triangulierungen, Enseign. Math. (2) 38 (1992) 201-217 MR1189006

[3] B Chow, F Luo, Combinatorial Ricci flows on surfaces, J. Differential Geom. 63 (2003) 97-129 MR2015261

[4] Y Colin de Verdière, Un principe variationnel pour les empilements de cercles, Invent. Math. 104 (1991) 655-669 MR1106755

[5] W Fenchel, J Nielsen, Discontinuous groups of isometries in the hyperbolic plane, de Gruyter Studies in Math. 29, Walter de Gruyter \& Co., Berlin (2003) MR1958350 Edited and with a preface by A L Schmidt, Biography of the authors by B Fuglede

[6] R Guo, On parameterizations of Teichmüller spaces of surfaces with boundary, to appear in J. Differential Geom. arXiv:math.GT/0612221

[7] G P Hazel, Triangulating Teichmüller space using the Ricci flow, $\mathrm{PhD}$ thesis, University of California San Diego (2004) Available at www.math.ucsd.edu/ thesis/ thesis/ghazel/ghazel.pdf

[8] G Leibon, Characterizing the Delaunay decompositions of compact hyperbolic surfaces, Geom. Topol. 6 (2002) 361-391 MR1914573

[9] F Luo, Rigidity of polyhedral surfaces arXiv:math.GT/0612714

[10] F Luo, A characterization of spherical polyhedral surfaces, J. Differential Geom. 74 (2006) 407-424 MR2269784

[11] F Luo, On Teichmüller spaces of surfaces with boundary, Duke Math. J. 139 (2007) 463-482 MR2350850

[12] G Mondello, Triangulated Riemann surfaces with boundary and the Weil-Petersson Poisson structure, J. Differential Geom. 81 (2009) 391-436

[13] R C Penner, The decorated Teichmüller space of punctured surfaces, Comm. Math. Phys. 113 (1987) 299-339 MR919235

[14] I Rivin, Euclidean structures on simplicial surfaces and hyperbolic volume, Ann. of Math. (2) 139 (1994) 553-580 MR1283870

[15] J-M Schlenker, Circle patterns on singular surfaces, Discrete Comput. Geom. 40 (2008) 47-102 MR2429649

[16] K Stephenson, Introduction to circle packing: The theory of discrete analytic functions, Cambridge Univ. Press (2005) MR2131318

[17] W P Thurston, The geometry and topology of three-manifolds, Princeton Univ. Math. Dept. Lecture Notes (1979) Available at http://msri.org/publications/books/ gt $3 \mathrm{~m} /$ 
[18] W P Thurston, Three-dimensional geometry and topology. Vol. 1, Princeton Math. Series 35, Princeton University Press (1997) MR1435975 Edited by S Levy

School of Mathematics, University of Minnesota

Minneapolis, MN 55455, USA

The Center of Mathematical Science, Zhejiang University

Hangzhou, Zhejiang 310027, China

and

Department of Mathematics, Rutgers University

Piscataway, NJ 08854, USA

guoxx170@math.umn.edu, fluo@math.rutgers.edu

Proposed: David Gabai

Seconded: Peter Teichner, Walter Neumann

Received: 5 November 2007

Accepted: 17 January 2009 